Épijournal de Géométrie Algébrique

epiga.episciences.org

Volume 1 (2017), Article Nr. 9

\title{
Haas' Theorem revisited
}

\author{
Benoît Bertrand, Erwan Brugallé, and Arthur Renaudineau
}

\begin{abstract}
Haas' theorem describes all patchworkings of a given non-singular plane tropical curve $C$ giving rise to a maximal real algebraic curve. The space of such patchworkings is naturally a linear subspace $W_{C}$ of the $\mathbb{Z} / 2 \mathbb{Z}$-vector space $\vec{\Pi}_{C}$ generated by the bounded edges of $C$, and whose origin is the Harnack patchworking. The aim of this note is to provide an interpretation of affine subspaces of $\vec{\Pi}_{C}$ parallel to $W_{C}$.

To this purpose, we work in the setting of abstract graphs rather than plane tropical curves. We introduce a topological surface $S_{\Gamma}$ above a trivalent graph $\Gamma$, and consider a suitable affine space $\Pi_{\Gamma}$ of real structures on $S_{\Gamma}$ compatible with $\Gamma$. We identify the vector subspace $W_{\Gamma}$ of $\vec{\Pi}_{\Gamma}$ characterizing real structures inducing the same action on $H_{1}\left(S_{\Gamma}, \mathbb{Z} / 2 \mathbb{Z}\right)$. We then deduce from this statement another proof of Haas' original result.
\end{abstract}

Keywords. Patchworking; Viro method; Hilbert 16th problem; tropical curves; topology of involutions; maximal curves

2010 Mathematics Subject Classification. 14P25; $14 \mathrm{~T} 05$

\section{[Français]}

\section{Titre. Théorème de Haas revisité}

Résumé. Le théorème de Haas décrit tous les patchworks d'une courbe tropicale plane lisse donnée $C$ donnant lieu à une courbe algébrique réelle maximale. L'espace de ces patchworks est naturellement un sous-espace linéaire $W_{C}$ du $\mathbb{Z} / 2 \mathbb{Z}$-espace vectoriel $\vec{\Pi}_{C}$ engendré par les arêtes bornées de $C$, et dont l'origine est le patchwork de Harnack. Le but de cette note est de donner une interprétation des sous-espaces affines de $\vec{\Pi}_{C}$ parallèles à $W_{C}$.

Pour ce faire, nous nous plaçons dans le cadre des graphes abstraits plutôt que celui des courbes tropicales planes. Nous introduisons une surface topologique $S_{\Gamma}$ au dessus d'un graphe trivalent $\Gamma$, et considérons un espace affine adéquat $\Pi_{\Gamma}$ de structures réelles sur $S_{\Gamma}$ compatibles avec $\Gamma$. Nous identifions le sous-espace vectoriel $W_{\Gamma}$ de $\vec{\Pi}_{\Gamma}$ caractérisant des structures réelles induisant la même action sur $H_{1}\left(S_{\Gamma}, \mathbb{Z} / 2 \mathbb{Z}\right)$. Nous déduisons alors de cet énoncé une autre démonstration du résultat original de Haas.

Received by the Editors on September 14, 2016, and in final form on July 3, 2017.

Accepted on July 28, 2017.

\section{Benoît Bertrand}

Institut de mathématiques de Toulouse - IUT de Tarbes, 1 rue Lautréamont - CS 41624, 65016 Tarbes, France

e-mail: benoit.bertrand@math.univ-toulouse.fr

Erwan Brugallé

École polytechnique, CNRS, Université Paris-Saclay, 91128 Palaiseau Cedex, France

e-mail: erwan.brugalle@math.cnrs.fr

Arthur Renaudineau

Eberhard Karls Universität Tübingen, Fachbereich Mathematik, Institut für Geometrie, Germany

e-mail: arren@math.uni-tuebingen.de 


\section{Contents}

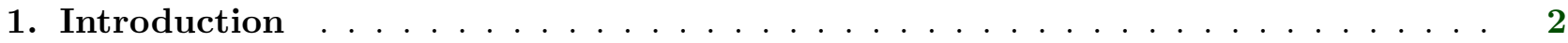

2. Real structures above real graphs $\ldots \ldots \ldots \ldots \ldots \ldots$

3. Action on $H_{1}\left(S_{\Gamma} ; \mathbb{Z} / 2 \mathbb{Z}\right)$ induced by a real structure $\ldots \ldots \ldots \ldots$

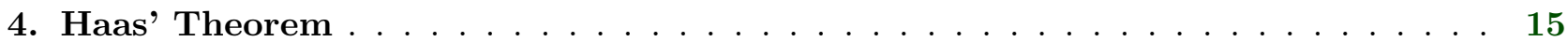

\section{Introduction}

Haas classified in his thesis [Haa97] all unimodular combinatorial patchworkings (see [Vir84, IV96]) producing a real algebraic $M$-curve (i.e. a non-singular compact real algebraic curve of genus $g$ whose real part has $g+1$ connected components). In the tropical reformulation of patchworking in terms of twist-admissible sets of edges of a non-singular plane tropical curve $C$ given in [BIMS15], the set of all possible patchworkings with this given underlying tropical curve $C$ is naturally a vector space $\vec{\Pi}_{C}$ over $\mathbb{Z} / 2 \mathbb{Z}$ and Haas' Theorem can be interpreted as follows: the space of all such patchworkings producing an $M$-curve is a well identified and easily described subvector space $W_{C}$ of $\vec{\Pi}_{C}$ (see Section 4.A). In this note we address the question of interpreting affine subspaces of $\vec{\Pi}_{C}$ parallel to $W_{C}$. To

this purpose, it is suitable to follow Klein's approach and to work in the framework of abstract real topological surfaces, i.e. oriented topological surfaces equipped with an orientation-reversing continuous involution, rather than restricting to real algebraic curves in a given toric surface (see [Kle76]). Accordingly, we deal with abstract graphs rather than with plane tropical curves. Given an abstract graph $\Gamma$ with only 3 -valent and 1 -valent vertices, we construct a topological surface $S_{\Gamma}$ decomposed into a union of disks, cylinders, and pairs of pants (this is just a variation on standard pair of pants decompositions of a surface). Next, given a continuous involution $\tau: \Gamma \rightarrow \Gamma$, we define real structures above the pair $(\Gamma, \tau)$, which are roughly real structures on $S_{\Gamma}$ compatible with the decomposition induced by $\Gamma$ together with $\tau$ (see Section 2.C for precise definitions). The set of real structures above $(\Gamma, \tau)$ is naturally an affine space $\Pi_{(\Gamma, \tau)}$ over $\mathbb{Z} / 2 \mathbb{Z}$ whose direction $\vec{\Pi}_{(\Gamma, \tau)}$ has for basis the set of edges of the quotient graph $\Gamma / \tau$ that are adjacent to two 3 -valent vertices (see Lemma 2.11). As any involution, a real structure on $S_{\Gamma}$ induces an action on $H_{1}\left(S_{\Gamma} ; \mathbb{Z} / 2 \mathbb{Z}\right)$ and the main result of this note can be summarized as follows.

Two real structures above $(\Gamma, \tau)$ induce the same action on $H_{1}\left(S_{\Gamma} ; \mathbb{Z} / 2 \mathbb{Z}\right)$ if and only if they differ by an element of a given vector subspace $W_{(\Gamma, \tau)}$ of $\overrightarrow{\Pi_{(\Gamma, \tau)}}$ having a simple description.

This statement is proved in Theorem 3.2 after the definition of $W_{(\Gamma, \tau)}$ at the begining of Section 3 . We show in Proposition 3.5 that $W_{(\Gamma, \tau)}$ admits an alternative description which corresponds to Haas' description of the above-mentioned vector space $W_{C}$ in the special case of a non-singular plane tropical curve $C$. The connection of Theorem 3.2 and Proposition 3.5 with Haas' Theorem then comes from the fact that a real topological surface with a non-empty real part is maximal if and only if the corresponding induced action on the first homology group of the surface is trivial. In particular, we recover Haas' Theorem as a corollary of our main results combined with standard results in tropical geometry (see Section 4.B).

Our main motivation for the present work was the possible generalisations of Haas' Theorem in higher dimensions. Generalising a statement usually first requires to identify the suitable notions coming into play and allowing a suitable formulation of the original statement. Both in its original formulation and in its "twist-admissible" tropical reformulation, Haas' Theorem involves, sometimes implicitely, several features that a priori make sense only for curves. As an example, it is based 
on the existence of Harnack distribution of signs discovered by Itenberg (see [IV96]), which has no known analogue yet in higher dimensions. The existence of such Harnack distribution of signs on $\mathbb{Z}^{2}$ is precisely the fact that naturally turns the space of all patchworkings of a non-singular tropical curve in $\mathbb{R}^{2}$ in a vector space rather than an affine space as in the case of abstract graphs: it provides a canonical patchworking on any non-singular tropical curve in $\mathbb{R}^{2}$, which in addition produces an $M$-curve. By focusing on abstract graphs rather than on embedded tropical curves, and on the action on homology induced by a real structure rather than the number of connected components of its real part, we place Haas' Theorem in a wider perspective. There, it turns out to be a corollary of a more general statement which seems to us more likely to have a higher dimensional analogue.

\section{A few words on the context}

Despite the surprising elegance of the complete description of maximal unimodular patchworkings, Haas' Theorem unfortunately only ever appeared in his thesis [Haa97]. We briefly recap its origin, rooted in the first part of Hilbert's 16th problem. This latter concerns the classification, up to isotopy, of all possible mutual positions of the connected components of the real part of a non-singular real algebraic curve in $\mathbb{R P}^{2}$ of a given degree $d$. If $d$ is even, every connected component of the real part of such a curve is called an oval, and bounds a disc which is called the interior of the oval. One says that an oval is even (resp. odd) if it is contained in the interior of an even (resp. odd) number of ovals. The following Ragsdale conjecture played an important role in subsequent developments in real algebraic geometry, and remains one challenging open question in the case of $M$-curves.

Conjecture (Refined Ragsdale conjecture, [Rag06, Pet33]). For any non-singular real algebraic curve of even degree $2 k$ in $\mathbb{R P}^{2}$ having $p$ even and $n$ odd ovals, one has

$$
p \leq \frac{3 k(k-1)}{2}+1 \quad \text { and } \quad n \leq \frac{3 k(k-1)}{2}+1 .
$$

A series of counterexamples to this conjecture have been constructed since the 90's [Ite93, Haa95, Bru06]. Nevertheless, no counterexample is known yet among $M$-curves. As an application of his classification of maximal unimodular combinatorial patchworkings, Haas proved in his thesis the following theorem.

Theorem (Haas, [Haa97, Theorem 12.4.0.12 and Proposition 13.5.0.13]). Let $A$ be a non-singular real algebraic $M$-curve in $\mathbb{R P}^{2}$ constructed by a unimodular combinatorial patchworking. If $A$ has $p$ even and $n$ odd ovals, then one has

$$
p \leq \frac{3 k(k-1)}{2}+1 \quad \text { and } \quad n \leq \frac{3 k(k-1)}{2}+4 .
$$

Furthermore, such a curve having more than $\frac{3 k(k-1)}{2}+1$ odd ovals would have exactly $n=$ $\frac{3 k(k-1)}{2}+4$ such ovals.

As far as we know, this is the only known result in the direction of Ragsdale conjecture for maximal curves.

\section{Organisation of the paper}

We start by defining real structures above an abstract graph in Section 2. In Section 3, we prove our main statement (Theorem 3.2) and Proposition 3.5 which relates our definition of $W_{(\Gamma, \tau)}$ to Haas' description of the vector space $W_{C}$. We end this paper by recalling in Section 4 the combinatorial patchworking construction and Haas' Theorem, and by deducing this latter from results from Section 3. 


\section{Acknowledgements}

Part of this work has been done during visits of B.B. and A.R. at Centre Mathématiques Laurent Schwartz, École Polytechnique. We thank this institution for excellent working conditions. We also thank Ilia Itenberg for fruitful discussions, as well as anonymous referees for many valuable comments on an earlier version of this paper.

\section{Convention and notation}

A real topological surface $(S, \tau)$ is an oriented topological surface $S$ equipped with an orientationreversing continuous involution $\tau: S \rightarrow S$, called a real structure. Given a real surface $(S, \tau)$, we denote by $\mathbb{R} S$ the real part of $S$, i.e. the set of fixed points of $\tau$. It is a submanifold of $S$ of dimension 1. If $S$ is compact and of genus $g$, then $\mathbb{R} S$ has at most $g+1$ connected components (see [Kle76]) and we say that $(S, \tau)$ is maximal if it has $g+1$ connected components. If $(S, \tau)$ is a compact real surface and $A \subset S$ is a real finite set of points, we say that $(S \backslash A, \tau)$ is maximal if $(S, \tau)$ is.

Given a finite graph $\Gamma$, we denote by $\operatorname{Vert}(\Gamma)$ the set of its vertices, by $\operatorname{Vert}^{\infty}(\Gamma)$ the set of its 1 -valent vertices, and we set $\operatorname{Vert}^{0}(\Gamma)=\operatorname{Vert}(\Gamma) \backslash \operatorname{Vert}^{\infty}(\Gamma)$. By definition, the valency of a vertex $v \in \operatorname{Vert}(\Gamma)$, denoted by $\operatorname{val}(v)$, is the number of edges of $\Gamma$ adjacent to $v$. We also denote by Edge $(\Gamma)$ the set of edges of $\Gamma$, and by $\operatorname{Edge}^{0}(\Gamma)$ the set of edges of $\Gamma$ adjacent to two vertices in $\operatorname{Vert}^{0}(\Gamma)$. Throughout the text we identify a graph with any of its topological realisations in which edges are open segments.

\section{Real structures above real graphs}

\section{A. Surfaces associated to a trivalent graph}

We will call trivalent a graph such that any of its vertices is either 3-valent or 1-valent, thus authorizing that it also has leaves. Given a trivalent graph $\Gamma$, we construct a topological surface $S_{\Gamma}$ as follows. Recall that a pair of pants is an oriented sphere with three open disks removed, in particular it has three boundary components with induced orientation.

1. To each vertex $v$ of $\Gamma$, we associate a topological surface $S_{v}$, which is either a pair of pants if $v$ is 3 -valent, or an oriented closed disk if $v$ is 1 -valent. Furthermore, we choose a one to one correspondence between boundary components of $S_{v}$ and edges of $\Gamma$ adjacent to $v$; the boundary component corresponding to $e$ is denoted by $\gamma_{v, e}$.

2. To each edge $e$ of $\Gamma$, we associate an oriented cylinder $S_{e}$, and a one to one correspondence between boundary components of $S_{e}$ and vertices adjacent to $e$; the boundary component corresponding to $v$ is denoted by $\gamma_{e, v}$.

3. To each pair $(v, e) \in \operatorname{Vert}(\Gamma) \times \operatorname{Edge}(\Gamma)$ such that $e$ is adjacent to $v$, we associate an orientationreversing homeomorphism $\phi_{v, e}: \gamma_{v, e} \rightarrow \gamma_{e, v}$.

The surface $S_{\Gamma}$ is obtained by gluing all surfaces $S_{v}$ and $S_{e}$ via the maps $\phi_{v, e}$. It is a closed oriented topological surface. Clearly, the surface $S_{\Gamma}$ is not uniquely defined by $\Gamma$, but also depends on the choice of the homeomorphisms $\phi_{v, e}$ and of the surfaces $S_{v}$. However, different surfaces obtained by different choices are homeomorphic, and such an homeomorphism is canonical up to composition by Dehn twists along the circles $\gamma_{e, v}$ in $S_{\Gamma}$.

Example 2.1. We depicted in Figure 1 a trivalent graph $\Gamma$ and the corresponding surface $S_{\Gamma}$. 


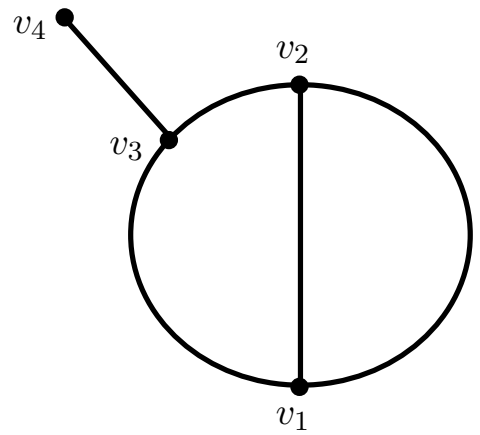

$\Gamma$

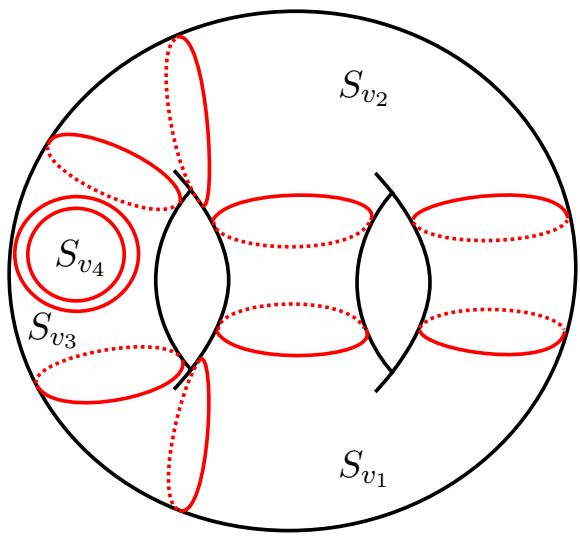

$S_{\Gamma}$

Figure 1: A trivalent graph $\Gamma$ and the corresponding surface $S_{\Gamma}$

\section{B. The groups $H_{1,0}\left(S_{\Gamma} ; \mathbb{Z} / 2 \mathbb{Z}\right)$ and $H_{0,1}\left(S_{\Gamma} ; \mathbb{Z} / 2 \mathbb{Z}\right)$}

Let $\Gamma$ be a trivalent graph. Each circle $\gamma_{e, v}$ defines an element in $H_{1}\left(S_{\Gamma} ; \mathbb{Z} / 2 \mathbb{Z}\right)$. If $v$ and $v^{\prime}$ are the two vertices adjacent to $e$, both circles $\gamma_{e, v}$ and $\gamma_{e, v^{\prime}}$ define the same element in $H_{1}\left(S_{\Gamma} ; \mathbb{Z} / 2 \mathbb{Z}\right)$, that we denote by $\gamma_{e}$. We define $H_{1,0}\left(S_{\Gamma} ; \mathbb{Z} / 2 \mathbb{Z}\right)$ to be the subgroup of $H_{1}\left(S_{\Gamma} ; \mathbb{Z} / 2 \mathbb{Z}\right)$ generated by all the loops $\gamma_{e}$, and we set

$$
H_{0,1}\left(S_{\Gamma} ; \mathbb{Z} / 2 \mathbb{Z}\right)=H_{1}\left(S_{\Gamma} ; \mathbb{Z} / 2 \mathbb{Z}\right) / H_{1,0}\left(S_{\Gamma} ; \mathbb{Z} / 2 \mathbb{Z}\right) .
$$

Let $\alpha_{1}, \ldots, \alpha_{g}$ be a basis of $H_{1}(\Gamma ; \mathbb{Z} / 2 \mathbb{Z})$. To each 1 -cycle $\alpha_{i}$, we associate a class $\gamma_{\alpha_{i}}$ in $H_{1}\left(S_{\Gamma} ; \mathbb{Z} / 2 \mathbb{Z}\right)$ as follows:

1. for each $e \in \operatorname{Edge}(\Gamma)$ such that $e \subset \alpha_{i}$, choose a 1-chain $\eta_{e}$ in $S_{e}$ joining the two boundary components of $S_{e}$. Denote by $\eta_{v, e}$ the boundary point of $\eta_{e}$ contained in $\gamma_{e, v}$;

2. for each $v \in \operatorname{Vert}(\Gamma)$ such that $v \in \alpha_{i}$, choose a 1-chain $\eta_{v}$ in $S_{v}$ joining the points $\eta_{v, e}$ and $\eta_{v, e^{\prime}}$, where $e$ and $e^{\prime}$ are the two edges contained in $\alpha_{i}$ and adjacent to $v$.

We denote by $\gamma_{\alpha_{i}} \in H_{1}\left(S_{\Gamma} ; \mathbb{Z} / 2 \mathbb{Z}\right)$ the class defined by the union of all 1-chains $\eta_{e}$ and $\eta_{v}$ above. Further, we extend the map $\alpha_{i} \mapsto \gamma_{\alpha_{i}}$ by linearity to an injective $\mathbb{Z} / 2 \mathbb{Z}$-linear map

$$
\begin{array}{ccc}
H_{1}(\Gamma ; \mathbb{Z} / 2 \mathbb{Z}) & \longrightarrow & H_{1}\left(S_{\Gamma} ; \mathbb{Z} / 2 \mathbb{Z}\right) \\
\alpha & \longmapsto & \gamma_{\alpha}
\end{array} .
$$

Note that $\gamma_{\alpha}$ is not uniquely defined by $\alpha$, but is well defined only up to an element in $H_{1,0}\left(S_{\Gamma} ; \mathbb{Z} / 2 \mathbb{Z}\right)$. In other words, there is a natural and well defined injective $\mathbb{Z} / 2 \mathbb{Z}$-linear map (or group morphism)

$$
H_{1}(\Gamma ; \mathbb{Z} / 2 \mathbb{Z}) \longrightarrow H_{0,1}\left(S_{\Gamma} ; \mathbb{Z} / 2 \mathbb{Z}\right)
$$

that associates to $\alpha$ the class realised by $\gamma_{\alpha}$ in $H_{0,1}\left(S_{\Gamma} ; \mathbb{Z} / 2 \mathbb{Z}\right)$. The next lemma shows in particular that this map is an isomorphism.

Example 2.2. We consider the trivalent graph from Example 2.1. We depicted in Figure 2 the 1cycle $\gamma_{e}$, and two possible 1-cycles $\gamma_{\alpha}$, denoted by $\gamma_{\alpha}$ and $\gamma_{\alpha}^{\prime}$ (recall that the decomposition of $S_{\Gamma}$ into the union of the surfaces $S_{v}$ and $S_{e}$ is depicted in Figure 1).

Lemma 2.3. Let $\Gamma$ be a trivalent graph with $b_{1}(\Gamma)=g$. Then both $\mathbb{Z} / 2 \mathbb{Z}$-vector spaces $H_{1,0}\left(S_{\Gamma} ; \mathbb{Z} / 2 \mathbb{Z}\right)$ and $H_{0,1}\left(S_{\Gamma} ; \mathbb{Z} / 2 \mathbb{Z}\right)$ have dimension $g$. Furthermore, the intersection form on $H_{1}\left(S_{\Gamma} ; \mathbb{Z} / 2 \mathbb{Z}\right)$ vanishes on $H_{1,0}\left(S_{\Gamma} ; \mathbb{Z} / 2 \mathbb{Z}\right)$. 


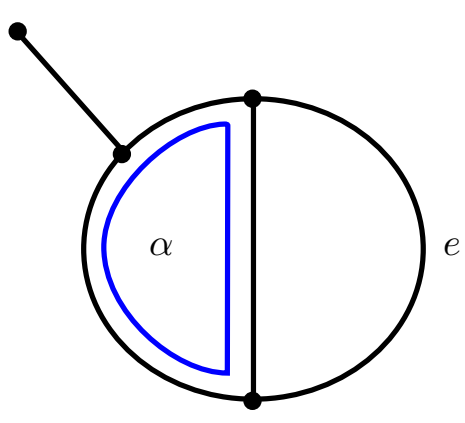

$\Gamma$

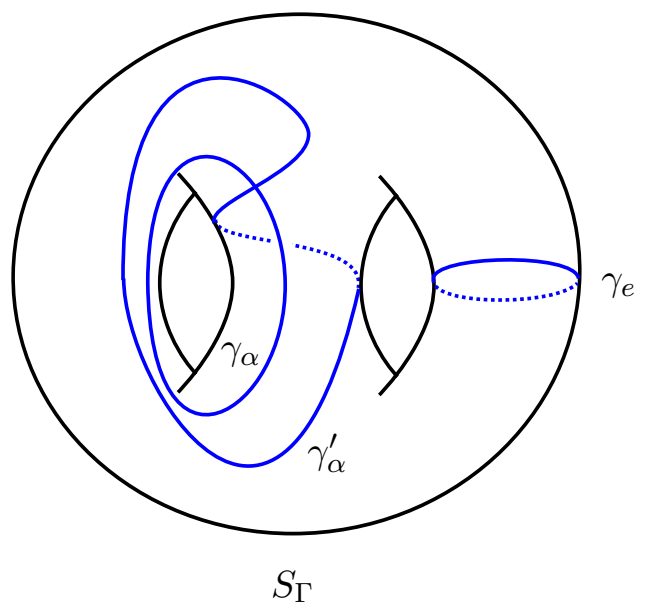

$S_{\Gamma}$

Figure 2: Lifting cycles

Proof. The group $H_{1,0}\left(S_{\Gamma} ; \mathbb{Z} / 2 \mathbb{Z}\right)$ is clearly contained in its orthogonal for the intersection form on $H_{1}\left(S_{\Gamma} ; \mathbb{Z} / 2 \mathbb{Z}\right)$. Since this latter is non-degenerate, we deduce that $H_{1,0}\left(S_{\Gamma} ; \mathbb{Z} / 2 \mathbb{Z}\right)$ has dimension at most $g$. Let $U$ be the vector subspace of $H_{1}\left(S_{\Gamma} ; \mathbb{Z} / 2 \mathbb{Z}\right)$ of dimension $g$ generated by all classes $\gamma_{\alpha}$ with $\alpha \in H_{1}(\Gamma ; \mathbb{Z} / 2 \mathbb{Z})$. By construction, the intersection of $U$ with the orthogonal of $H_{1,0}\left(S_{\Gamma} ; \mathbb{Z} / 2 \mathbb{Z}\right)$ is trivial, from which we deduce that $H_{1,0}\left(S_{\Gamma} ; \mathbb{Z} / 2 \mathbb{Z}\right)$ has dimension at least $g$. Hence $H_{1,0}\left(S_{\Gamma} ; \mathbb{Z} / 2 \mathbb{Z}\right)$ has dimension $g$. Since by definition, the group $H_{0,1}\left(S_{\Gamma} ; \mathbb{Z} / 2 \mathbb{Z}\right)$ is the quotient of $H_{1}\left(S_{\Gamma} ; \mathbb{Z} / 2 \mathbb{Z}\right)$ by $H_{1,0}\left(S_{\Gamma} ; \mathbb{Z} / 2 \mathbb{Z}\right)$, it also has dimension $g$.

We will abusively identify $H_{0,1}\left(S_{\Gamma} ; \mathbb{Z} / 2 \mathbb{Z}\right)$ with the subgroup of $H_{1}\left(S_{\Gamma} ; \mathbb{Z} / 2 \mathbb{Z}\right)$ generated by all classes $\gamma_{\alpha}$ with $\alpha \in H_{1}(\Gamma ; \mathbb{Z} / 2 \mathbb{Z})$. We then have the decomposition

$$
H_{1}\left(S_{\Gamma} ; \mathbb{Z} / 2 \mathbb{Z}\right)=H_{1,0}\left(S_{\Gamma} ; \mathbb{Z} / 2 \mathbb{Z}\right) \oplus H_{0,1}\left(S_{\Gamma} ; \mathbb{Z} / 2 \mathbb{Z}\right) .
$$

Hence the group $H_{0,1}\left(S_{\Gamma} ; \mathbb{Z} / 2 \mathbb{Z}\right)$ viewed as a subgroup of $H_{1}\left(S_{\Gamma} ; \mathbb{Z} / 2 \mathbb{Z}\right)$ is the image of a section of the quotient map $H_{1}\left(S_{\Gamma} ; \mathbb{Z} / 2 \mathbb{Z}\right) \rightarrow H_{0,1}\left(S_{\Gamma} ; \mathbb{Z} / 2 \mathbb{Z}\right)$.

Remark 2.4. Lemma 2.3 and the filtration of $H_{1}\left(S_{\Gamma} ; \mathbb{Z} / 2 \mathbb{Z}\right)$ by $H_{1,0}\left(S_{\Gamma} ; \mathbb{Z} / 2 \mathbb{Z}\right)$ and $H_{0,1}\left(S_{\Gamma} ; \mathbb{Z} / 2 \mathbb{Z}\right)$ may be seen as very particular cases of [IKMZ16, Theorem 1]. This relation to tropical homology explains our choice of notation.

\section{C. Real trivalent graphs and real structures above them}

Definition 2.5. A real trivalent graph is a pair $(\Gamma, \tau)$, where $\Gamma$ is a trivalent graph and $\tau: \Gamma \rightarrow \Gamma$ a continuous involution (called real structure), such that the restriction of $\tau$ on any (open) edge of $\Gamma$ is either the identity or has no fixed points.

The condition on the restriction of $\tau$ on edges of $\Gamma$ is to exclude the situation when this restriction is a symmetry locally given by $x \mapsto-x$.

Example 2.6. Any trivalent graph $\Gamma$ carries a canonical real structure given by $\tau=\mathrm{Id}$.

Example 2.7. Two real trivalent graphs $(\Gamma, \tau)$ are depicted in Figure 3. The graph on the left is of genus 2 whereas the other one is of genus 3 . In both cases the graph $\Gamma$ is drawn on the plane, and $\tau$ is the axial symmetry with respect to the line supporting edges $e_{1}$ (resp. $e_{1}$ and $e_{2}$ ). Hence $e_{1}$ (resp. $e_{1}$ and $e_{2}$ ) are exactly the $\tau$-invariant edges of $\Gamma$. 


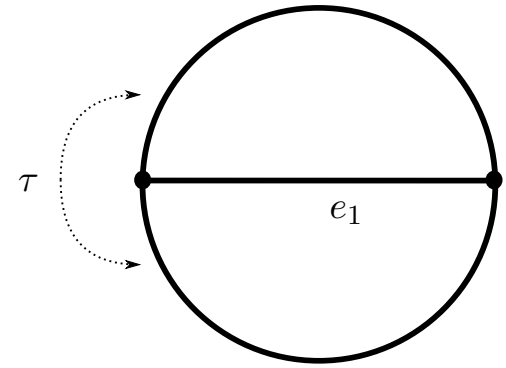

a) A real trivalent graph $(\Gamma, \tau)$ of genus 2

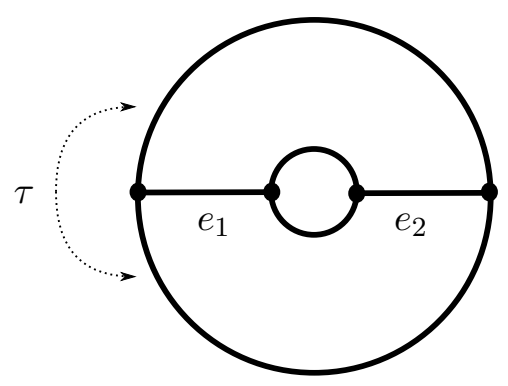

b) A real trivalent graph $(\Gamma, \tau)$ of genus 3

Figure 3: Real graphs

Next we define the lifts of $\tau$ to $S_{\Gamma}$ that we consider in this text. For this purpose, we need to fix the following:

- the closed disk $\mathcal{S}_{1}=\{z \in \mathbb{C},|z| \leq 1\}$ equipped with the complex conjugation $\sigma_{1}: \mathcal{S}_{1} \rightarrow \mathcal{S}_{1}$;

- a pair of pants $\mathcal{S}_{3}$;

- two orientation-reversing involutions $\sigma_{3}: \mathcal{S}_{3} \rightarrow \mathcal{S}_{3}$ and $\widetilde{\sigma}: \mathcal{S}_{3} \rightarrow \mathcal{S}_{3}$ such that the fixed locus of $\sigma_{3}$ is three disjoint segments, and the fixed locus of $\widetilde{\sigma}$ is a segment (see Figure 4);
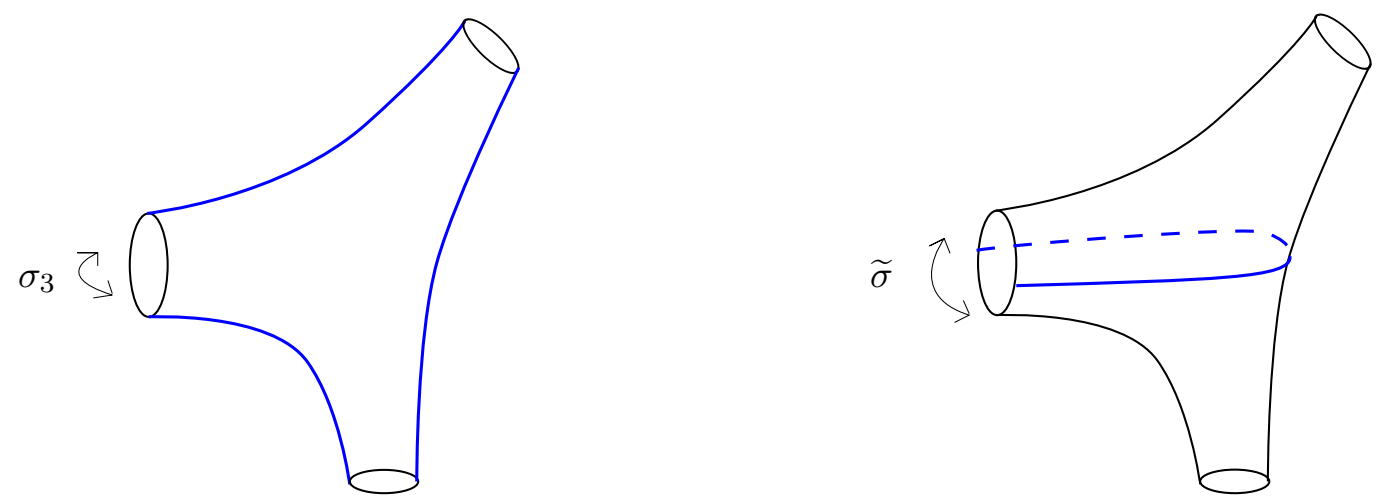

Figure 4: Two real structures on a pair of pants

- for each vertex $v$ of $\Gamma$, an orientation-preserving homeomorphism $\psi_{v}: S_{v} \rightarrow \mathcal{S}_{\mathrm{val}(v)}$; if in addition $\tau$ interchanges two adjacent edges of a $\tau$-invariant vertex $v$, then $\psi_{v}$ is chosen so that $\tilde{\sigma}\left(\psi_{v}\left(\gamma_{v, e}\right)\right)=\psi_{v}\left(\gamma_{v, \tau(e)}\right)$ for any edge $e$ adjacent to $v$.

We say that a continuous orientation-reversing involution $\tau_{\Gamma}: S_{\Gamma} \rightarrow S_{\Gamma}$ lifts $\tau: \Gamma \rightarrow \Gamma$ if the following conditions are satisfied:

1. if $\tau$ is locally the identity around $v$, then $\left.\tau_{\Gamma}\right|_{S_{v}}=\psi_{v}^{-1} \circ \sigma_{\operatorname{val}(v)} \circ \psi_{v}$;

2. if $v$ is a 3 -valent vertex fixed by $\tau$, and if $\tau$ interchanges two adjacent edges of $v$, then $\left.\tau_{\Gamma}\right|_{S_{v}}=$ $\psi_{v}^{-1} \circ \tilde{\sigma} \circ \psi_{v}$

3. if $\tau(v) \neq v$, then $\left.\tau_{\Gamma}\right|_{S_{v}}=\psi_{\tau(v)}^{-1} \circ \sigma_{\operatorname{val}(v)} \circ \psi_{v}$. 
Note that the three above conditions imply that if $\tau_{\Gamma}$ lifts $\tau$, then $\tau_{\Gamma}\left(S_{e}\right)=S_{\tau(e)}$ for each edge of $\Gamma$.

We denote by $G$ the subgroup of the group of homeomorphisms of $S_{\Gamma}$ generated by elements $h: S_{\Gamma} \rightarrow S_{\Gamma}$ such that

- $h$ restricts to the identity on $\bigcup_{v \in \operatorname{Vert}^{0}(\Gamma)} S_{v}$;

- if $e$ is a $\tau$-invariant edge of $\Gamma$, then the restriction of $h$ on $S_{e}$ is isotopic to a power of the Dehn twist along $\gamma_{e}$;

- if $\tau(e) \neq e$, then the restriction of $h$ on $S_{e} \cup S_{\tau(e)}$ is isotopic to a power of the Dehn twist along $\gamma_{e}-\gamma_{\tau(e)}$.

Recall that by definition, two real topological surfaces $\left(S_{1}, \tau_{1}\right)$ and $\left(S_{2}, \tau_{2}\right)$ are isomorphic if there exists an orientation-preserving homeomorphism $\theta: S_{1} \rightarrow S_{2}$ such that $\theta \circ \tau_{1} \circ \theta^{-1}=\tau_{2}$. In particular, if $(\Gamma, \tau)$ is a real trivalent graph, and if two lifts $\tau_{\Gamma, 1}$ and $\tau_{\Gamma, 2}$ of $\tau$ are conjugated by an element of $G$, then the real topological surfaces $\left(S_{\Gamma}, \tau_{\Gamma_{1}}\right)$ and $\left(S_{\Gamma}, \tau_{\Gamma_{2}}\right)$ are isomorphic.

Definition 2.8. A real structure above a real trivalent graph $(\Gamma, \tau)$ is a lift of $\tau$ considered up to conjugation by an element of $G$.

Clearly, the set of lifts of $\tau$ for a real trivalent graph $(\Gamma, \tau)$ depends on all the choices made above. However, it follows from Proposition 2.11 below that the set of real structures above $(\Gamma, \tau)$ only depends on $(\Gamma, \tau)$.

Lemma 2.9. Let $\mathcal{S}_{2}$ be the cylinder $\{z \in \mathbb{C}|1 \leq| z \mid \leq 2\}$, and conj be the complex conjugation on $\mathbb{C}$. Denote by $T$ the Dehn twist of $\mathcal{S}_{2}$ along the circle of radius $\frac{3}{2}$ given by

$$
\begin{array}{ccc}
T: \mathcal{S}_{2} & \longrightarrow & \mathcal{S}_{2} \\
z & \longmapsto & e^{2 i \pi(|z|-1)} z
\end{array} .
$$

Then, up to isotopy and conjugation by a homeomorphism $\theta: \mathcal{S}_{2} \rightarrow \mathcal{S}_{2}$ restricting to the identity on $\partial \mathcal{S}_{2}$, the only real structures on $\mathcal{S}_{2}$ restricting to conj on $\partial \mathcal{S}_{2}$ are conj and $T \circ$ conj.

The real part of the two real structures conj and Toconj differ by a "half" Dehn twist, see Figure 5.
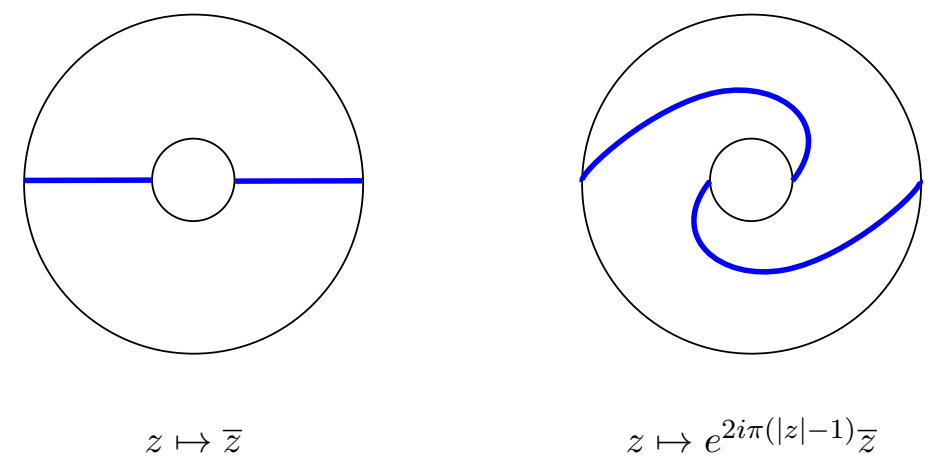

Figure 5: The real part of two real structures on the annulus $\{z \in \mathbb{C}|1 \leq| z \mid \leq 2\}$ 
Proof. Let $\tau_{1}: \mathcal{S}_{2} \rightarrow \mathcal{S}_{2}$ be an orientation-reversing continuous involution that restricts to conj on $\partial \mathcal{S}_{2}$. The map $\tau_{1}$ oconj is an orientation-preserving continuous involution that restricts to the identity on $\partial \mathcal{S}_{2}$. Hence it is, up to a composition by an isotopy restricting to the identity on $\partial \mathcal{S}_{2}$, equal to $T^{k}$ for some integer $k$. We compute

$$
T \circ \operatorname{conj} \circ T^{-1}(z)=e^{4 i \pi(|z|-1)} \bar{z}=T^{2} \circ \operatorname{conj} .
$$

Hence there exists a homeomorphism $\theta$ as in the lemma such that $\tau_{1}=\theta \circ \operatorname{conj} \circ \theta^{-1}$ if $k$ is even, and such that $\tau_{1}=\theta \circ T \circ$ conj $\circ \theta^{-1}$ if $k$ is odd.

If there would exist a homeomorphism $\theta$ as in the lemma such that conj $=\theta \circ T \circ \operatorname{conj} \circ \theta^{-1}$, it would map the real part of $T \circ$ conj to the real part of conj. Since these two real parts differ by a "half" Dehn twist, this is impossible.

Lemma 2.10. Let $\mathcal{S}_{2}^{\prime}$ be the cylinder $\left\{z \in \mathbb{C}\left|\frac{1}{4} \leq\right| z \mid \leq \frac{1}{2}\right\}$, let $\mathcal{S}_{2}^{\prime \prime}$ be the cylinder $\{z \in \mathbb{C}|2 \leq| z \mid \leq 4\}$. Denote respectively by $T_{1}$ and $T_{2}$ the Dehn twists of $\mathcal{S}_{2}^{\prime}$ and $\mathcal{S}_{2}^{\prime \prime}$ given by

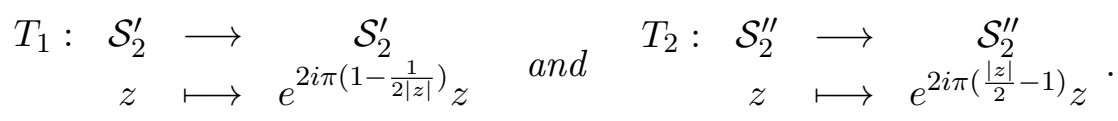

Then, up to isotopy and conjugation by a homeomorphism $\theta: \mathcal{S}_{2}^{\prime} \cup \mathcal{S}_{2}^{\prime \prime} \rightarrow \mathcal{S}_{2}^{\prime} \cup \mathcal{S}_{2}^{\prime \prime}$ restricting to the identity on $\partial\left(\mathcal{S}_{2}^{\prime} \cup \mathcal{S}_{2}^{\prime \prime}\right)$, the only real structures on $\mathcal{S}_{2}^{\prime} \cup \mathcal{S}_{2}^{\prime \prime}$ restricting to $\frac{1}{\text { conj }}$ on $\partial\left(\mathcal{S}_{2}^{\prime} \cup \mathcal{S}_{2}^{\prime \prime}\right)$ are $\frac{1}{\text { conj }}$ and $T_{1} \circ T_{2} \circ \frac{1}{\text { conj }}$.

Proof. The proof is similar to the proof of Lemma 2.9. Let $\tau_{1}: \mathcal{S}_{2}^{\prime} \cup \mathcal{S}_{2}^{\prime \prime}$ be an orientation-reversing continuous involution that restricts to $\frac{1}{\text { conj }}$ on $\partial\left(\mathcal{S}_{2}^{\prime} \cup \mathcal{S}_{2}^{\prime \prime}\right)$. The map $\tau_{1} \circ \frac{1}{\text { conj }}$ is an orientation-preserving continuous involution that restricts to the identity on $\partial\left(\mathcal{S}_{2}^{\prime} \cup \mathcal{S}_{2}^{\prime \prime}\right)$. Hence it is, up to a composition by an isotopy restricting to the identity on $\partial\left(\mathcal{S}_{2}^{\prime} \cup \mathcal{S}_{2}^{\prime \prime}\right)$, equal to $T_{1}^{k} \circ T_{2}^{l}$ for some integers $k$ and $l$. We extend the Dehn twists $T_{1}$ and $T_{2}$ to $\mathbb{C}$ by the identity respectively outside $\mathcal{S}_{2}^{\prime}$ and $\mathcal{S}_{2}^{\prime \prime}$. We compute

$$
\left(T_{1}^{k} \circ T_{2}^{l} \circ \frac{1}{\operatorname{conj}}\right)^{2}(z)=z e^{2 i \pi(l-k)\left(\frac{1}{2|z|}-1\right)}
$$

if $z \in \mathcal{S}_{2}^{\prime}$ and

$$
\left(T_{1}^{k} \circ T_{2}^{l} \circ \frac{1}{\text { conj }}\right)^{2}(z)=z e^{2 i \pi(l-k)\left(\frac{|z|}{2}-1\right)}
$$

if $z \in \mathcal{S}_{2}^{\prime \prime}$. Hence the map $\left(T_{1}^{k} \circ T_{2}^{l}\right) \circ \frac{1}{\text { conj }}$ is an involution on $\mathcal{S}_{2}^{\prime} \cup \mathcal{S}_{2}^{\prime \prime}$ if and only if $k=l$. We compute

$$
\left(T_{1} \circ T_{2}\right) \circ \frac{1}{\operatorname{conj}} \circ\left(T_{1}^{-1} \circ T_{2}^{-1}\right)=\left(T_{1}^{2} \circ T_{2}^{2}\right) \circ \frac{1}{\text { conj }},
$$

and the lemma is proved as Lemma 2.9.

The following proposition is the main observation that allows one to compare two different real structures above a given real trivalent graph $(\Gamma, \tau)$, or in other words, to make sense of the difference $\tau_{\Gamma, 1}-\tau_{\Gamma, 2}$ of two real structures in $\Pi_{(\Gamma, \tau)}$. Let $\Gamma / \tau$ be the quotient of the graph $\Gamma$ by $\tau$, i.e. edges and vertices exchanged by $\tau$ are identified.

Proposition 2.11. Let $(\Gamma, \tau)$ be a real trivalent graph. Let $e_{1}, \ldots, e_{k}$ be the edges in $E d g e^{0}(\Gamma / \tau)$, Then the set $\Pi_{(\Gamma, \tau)}$ of real structures above $(\Gamma, \tau)$ is naturally an affine space with direction

$$
\overrightarrow{\Pi_{(\Gamma, \tau)}}=\mathbb{Z} / 2 \mathbb{Z} e_{1} \oplus \mathbb{Z} / 2 \mathbb{Z} e_{2} \oplus \ldots \oplus \mathbb{Z} / 2 \mathbb{Z} e_{k} .
$$

Furthermore the set of fixed points of such a real structure $\tau_{\Gamma}$ is well defined up to isotopy and Dehn twists on cylinders corresponding to $\tau$-invariant edges in $E d g e^{0}(\Gamma)$. 
Proof. Let $e$ be a $\tau$-invariant edge in $\operatorname{Edge}^{0}(\Gamma)$. It follows from Lemma 2.9 that there exist exactly two possibilities for the restriction of a real structure on $S_{e}$, that are given, up to isotopy and conjugating by $T$, by conj and $T \circ$ conj on the cylinder $S_{e}=\{z \in \mathbb{C}|1 \leq| z \mid \leq 2\}$.

Similarly, for each pair of cylinders $\left\{S_{e}, S_{\tau(e)}\right\}$ with $e \in \operatorname{Edge}^{0}(\Gamma)$ such that $\tau(e) \neq e$, it follows from Lemma 2.10 that there are exactly two possibilities for the restriction of a real structure on $S_{e} \cup S_{\tau(e)}$, which differ by a composition with the Dehn twist along $\gamma_{e}-\gamma_{\tau(e)}$.

Example 2.12. On Figure 6 we show the fixed point set of two different real structures lifting the involution $\tau$ on the real graph of genus 2 depicted on Figure 3a.
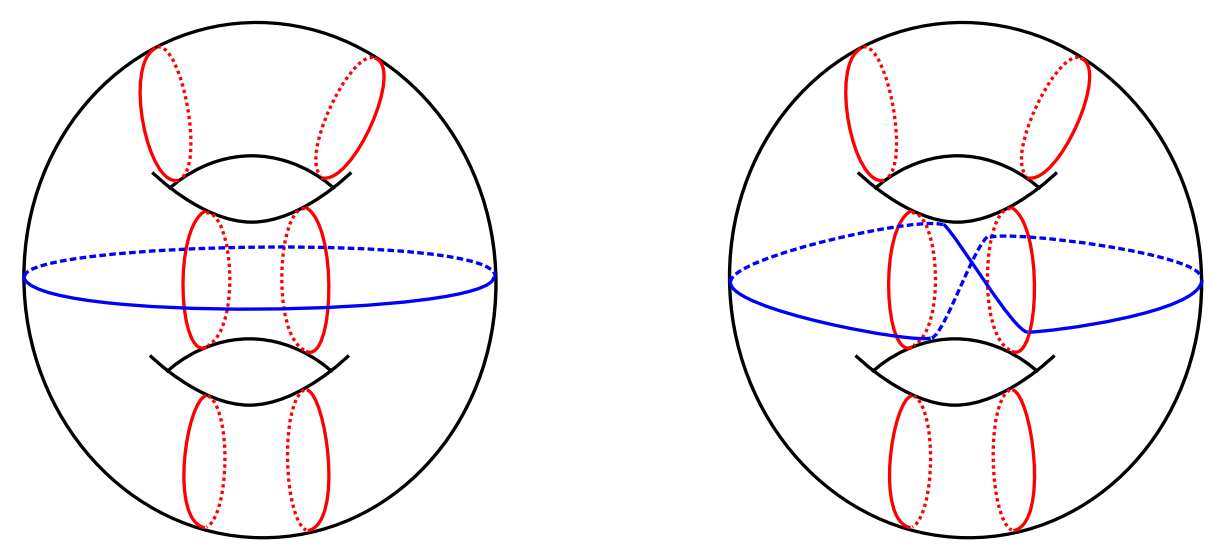

Figure 6: Two real structures above the same graph $(\Gamma, \tau)$ of genus 2 of Example 2.7

Remark 2.13. In the case when $\tau=\mathrm{Id}$, the data of a real structure $\tau_{\Gamma}: S_{\Gamma} \rightarrow S_{\Gamma}$ above $(\Gamma, \mathrm{Id})$ is equivalent to the data of the ribbon structure on $\Gamma$ given by $S_{\Gamma} / \tau_{\Gamma}$. Furthermore, two real structures differ along an edge $e$ of $\Gamma$ if and only if the two ribbon structures differ by a half twist along $e$.

\section{Action on $H_{1}\left(S_{\Gamma} ; \mathbb{Z} / 2 \mathbb{Z}\right)$ induced by a real structure}

We identify in this section a vector subspace $W_{(\Gamma, \tau)}$ of $\overrightarrow{\Pi_{(\Gamma, \tau)}}$ that characterises all real structures $\tau_{\Gamma}: S_{\Gamma} \rightarrow S_{\Gamma}$ inducing the same map $\tau_{\Gamma *}: H_{1}\left(S_{\Gamma} ; \mathbb{Z} / 2 \mathbb{Z}\right) \rightarrow H_{1}\left(S_{\Gamma} ; \mathbb{Z} / 2 \mathbb{Z}\right)$. Next lemma ensures that the action on $H_{1}\left(S_{\Gamma} ; \mathbb{Z} / 2 \mathbb{Z}\right)$ induced by a real structure above a real trivalent graph is well defined.

Lemma 3.1. Let $(\Gamma, \tau)$ be a real trivalent graph and $\tau_{\Gamma}: S_{\Gamma} \rightarrow S_{\Gamma}$ be a lift of $\tau$. Then the induced involution $\tau_{\Gamma *}: H_{1}\left(S_{\Gamma} ; \mathbb{Z} / 2 \mathbb{Z}\right) \rightarrow H_{1}\left(S_{\Gamma} ; \mathbb{Z} / 2 \mathbb{Z}\right)$ only depends on the real structure class of $\tau_{\Gamma}$.

Proof. It follows from the proof of Lemmas 2.9 and 2.10 that any other representative of the real structure class of $\tau_{\Gamma}$ is obtained from this latter by a finite sequence of compositions with either an isotopy or by an even power of a Dehn twist. Since both types of maps induce a trivial action on $H_{1}\left(S_{\Gamma} ; \mathbb{Z} / 2 \mathbb{Z}\right)$, the result follows.

Given $(\Gamma, \tau)$ a real trivalent graph, we denote by $\pi: \Gamma \rightarrow \Gamma / \tau$ the quotient map. Recall that by Proposition 2.11, an edge in $\operatorname{Edge}^{0}(\Gamma)$ defines a vector in $\overrightarrow{\Pi_{(\Gamma, \tau)}}$. There is a natural bilinear map

$$
\mu: H_{1}(\Gamma ; \mathbb{Z} / 2 \mathbb{Z}) \times H_{1}(\Gamma ; \mathbb{Z} / 2 \mathbb{Z}) \longrightarrow \overrightarrow{\Pi_{(\Gamma, \tau)}}
$$

that associates to two 1-cycles $\alpha$ and $\beta$ the sum of the vectors in $\overrightarrow{\Pi_{(\Gamma, \tau)}}$ defined by the edges contained in the support of the 1-chain $\pi(\alpha \cap \beta)$. We denote by $\langle.,$.$\rangle the standard bilinear form on \overrightarrow{\Pi_{(\Gamma, \tau)}}$ defined 
by

$$
\left\langle\sum_{e \in \operatorname{Edge}^{0}(\Gamma / \tau)} u_{e} e, \sum_{e \in \operatorname{Edge}^{0}(\Gamma / \tau)} v_{e} e\right\rangle=\sum_{e \in \operatorname{Edge}^{0}(\Gamma / \tau)} u_{e} v_{e} .
$$

We define the following vector subspace $W_{(\Gamma, \tau)}$ of $\overrightarrow{\Pi_{(\Gamma, \tau)}}$ :

$$
W_{(\Gamma, \tau)}=\left\{w \in \overrightarrow{\Pi_{(\Gamma, \tau)}} \mid \forall \alpha, \beta \in H_{1}(\Gamma ; \mathbb{Z} / 2 \mathbb{Z}),\langle w, \mu(\alpha, \beta)\rangle=0\right\} .
$$

By definition, we have

$$
W_{(\Gamma, \tau)}=\bigcap_{\alpha, \beta \in H_{1}(\Gamma ; \mathbb{Z} / 2 \mathbb{Z})} \operatorname{Ker}\left(\mu^{*}(\alpha, \beta)\right)
$$

where $\mu^{*}(\alpha, \beta)$ denotes the linear map dual to the vector $\mu(\alpha, \beta)$.

Theorem 3.2. Let $(\Gamma, \tau)$ be a real trivalent graph, and let $\tau_{\Gamma, 1}: S_{\Gamma} \rightarrow S_{\Gamma}$ and $\tau_{\Gamma, 2}: S_{\Gamma} \rightarrow S_{\Gamma}$ be two real structures above $(\Gamma, \tau)$. Then we have

$$
\tau_{\Gamma, 1 *}=\tau_{\Gamma, 2 *} \Longleftrightarrow \tau_{\Gamma, 1}-\tau_{\Gamma, 2} \in W_{(\Gamma, \tau)} .
$$

Proof. Clearly, both maps $\tau_{\Gamma, 1 *}$ and $\tau_{\Gamma, 2 *}$ have the same restriction to $H_{1,0}\left(S_{\Gamma} ; \mathbb{Z} / 2 \mathbb{Z}\right)$. Hence according to Lemma 2.3, we are left to show that

$$
\forall \alpha \in H_{1}(\Gamma ; \mathbb{Z} / 2 \mathbb{Z}), \quad \tau_{\Gamma, 1 *}\left(\gamma_{\alpha}\right)=\tau_{\Gamma, 2 *}\left(\gamma_{\alpha}\right) \Longleftrightarrow \tau_{\Gamma, 1}-\tau_{\Gamma, 2} \in W_{(\Gamma, \tau)} .
$$

Given a vector $w \in \overrightarrow{\Pi_{(\Gamma, \tau)}}$, we denote by $\mathcal{E}(w)$ the set of edges $e \in \operatorname{Edge}^{0}(\Gamma)$ such that $\langle w, \pi(e)\rangle=1$. Choosing other representatives of the real structure class of $\tau_{\Gamma, 1}$ and $\tau_{\Gamma, 2}$ if necessary, we may assume that these latter coincide on

$$
S_{\Gamma} \backslash \bigcup_{e \in \mathcal{E}\left(\tau_{\Gamma, 1}-\tau_{\Gamma, 2}\right)} S_{e}
$$

and differ by a composition with a Dehn twist along $\gamma_{e}$ for all edges $e$ in $\mathcal{E}\left(\tau_{\Gamma, 1}-\tau_{\Gamma, 2}\right)$. Hence we have

$$
\tau_{\Gamma, 1 *}\left(\gamma_{\alpha}\right)=\tau_{\Gamma, 2 *}\left(\gamma_{\alpha}\right)+\sum_{e \in \mathcal{E}\left(\tau_{\Gamma, 1}-\tau_{\Gamma, 2}\right) \cap \alpha} \gamma_{e}
$$

Since the intersection form is non-degenerate on $H_{1}\left(S_{\Gamma} ; \mathbb{Z} / 2 \mathbb{Z}\right)$, we have

$$
\tau_{\Gamma, 1 *}\left(\gamma_{\alpha}\right)-\tau_{\Gamma, 2 *}\left(\gamma_{\alpha}\right)=0 \Longleftrightarrow \forall \gamma \in H_{1}\left(S_{\Gamma} ; \mathbb{Z} / 2 \mathbb{Z}\right),\left(\tau_{\Gamma, 1 *}\left(\gamma_{\alpha}\right)-\tau_{\Gamma, 2 *}\left(\gamma_{\alpha}\right)\right) \cdot \gamma=0,
$$

where $a \cdot b \in \mathbb{Z} / 2 \mathbb{Z}$ stands for the intersection product of two classes $a$ and $b$ in $H_{1}\left(S_{\Gamma} ; \mathbb{Z} / 2 \mathbb{Z}\right)$. Combining Relation (3.1) and Lemma 2.3, we obtain

$$
\tau_{\Gamma, 1 *}\left(\gamma_{\alpha}\right)-\tau_{\Gamma, 2 *}\left(\gamma_{\alpha}\right)=0 \Longleftrightarrow \forall \beta \in H_{1}(\Gamma ; \mathbb{Z} / 2 \mathbb{Z}), \sum_{e \in \mathcal{E}\left(\tau_{\Gamma, 1}-\tau_{\Gamma, 2}\right) \cap \alpha} \gamma_{e} \cdot \gamma_{\beta}=0 .
$$

By construction we have

$$
\sum_{e \in \mathcal{E}\left(\tau_{\Gamma, 1}-\tau_{\Gamma, 2}\right) \cap \alpha} \gamma_{e} \cdot \gamma_{\beta}=\left\langle\tau_{\Gamma, 1}-\tau_{\Gamma, 2}, \mu(\alpha, \beta)\right\rangle,
$$

so the result is proved. 
Example 3.3. Let us consider the real trivalent graph $(\Gamma, \tau)$ of genus 3 depicted in Figure $3 \mathrm{~b}$, with two $\tau$-invariant edges $e_{1}$ and $e_{2}$. There exists 16 real structures above $(\Gamma, \tau)$, distributed into 8 parallel affine subspaces of $\Pi_{(\Gamma, \tau)}$ with direction $W_{(\Gamma, \tau)}=\mathbb{Z} / 2 \mathbb{Z} \pi\left(e_{1}+e_{2}\right)$. Note that the real part of the real topological surface $\left(S_{\Gamma}, \tau_{\Gamma}\right)$ is composed of two circles for any element $\tau_{\Gamma}$ of $\Pi_{(\Gamma, \tau)}$. Furthermore, all possible real structures above $(\Gamma, \tau)$ produce exactly two real isomorphism types of real topological surfaces: $\left(S_{\Gamma}, \tau_{\Gamma}\right)$ is of type ${ }^{1}$ I for 4 out of these 8 families of real structures, and of type II for the 4 remaining families.

Corollary 3.4. Let $(\Gamma, \tau)$ be a real trivalent graph, and let $\tau_{\Gamma, 1}: S_{\Gamma} \rightarrow S_{\Gamma}$ and $\tau_{\Gamma, 2}: S_{\Gamma} \rightarrow S_{\Gamma}$ be two real structures above $(\Gamma, \tau)$. Assume that $\left(S_{\Gamma}, \tau_{\Gamma, 1}\right)$ is a maximal real topological surface. Then $\left(S_{\Gamma}, \tau_{\Gamma, 2}\right)$ is also maximal if and only if the difference $\tau_{\Gamma, 1}-\tau_{\Gamma, 2}$ lies in $W_{(\Gamma, \tau)}$.

Proof. Recall that a real topological surface $\left(S, \tau_{S}\right)$ with $\mathbb{R} S \neq \emptyset$ is maximal if and only if $\tau_{S *}=\mathrm{Id}$, see [BR90, Proposition 5.4.9]. Hence we have $\tau_{\Gamma, 1 *}=$ Id. Furthermore, since $\left(S_{\Gamma}, \tau_{\Gamma, 1}\right)$ has a non-empty real part, the trivalent graph $\Gamma$ has at least one $\tau$-invariant edge, which in its turn implies that any real structure above $(\Gamma, \tau)$ has a non-empty real part. Then, by Theorem $3.2, \tau_{\Gamma, 2 *}=$ Id if and only if $\tau_{\Gamma, 1}-\tau_{\Gamma, 2} \in W_{(\Gamma, \tau)}$, that is to say $\left(S_{\Gamma}, \tau_{\Gamma, 2}\right)$ is maximal if and only if $\tau_{\Gamma, 1}-\tau_{\Gamma, 2}$ lies in $W_{(\Gamma, \tau)}$.

Next proposition gives an alternative description of the space $W_{(\Gamma, \tau)}$ in terms of disconnecting edges and disconnecting pairs of edges. Recall that edges are always considered open. Given a real connected trivalent graph $(\Gamma, \tau)$, we define the following sets:

- Edge $^{0,(1)}(\Gamma, \tau)$ is the set of disconnecting edges of $\Gamma / \tau$ in $\operatorname{Edge}^{0}(\Gamma / \tau)$, i.e. edges $e \in \operatorname{Edge}^{0}(\Gamma / \tau)$ such that $\Gamma / \tau \backslash e$ is not connected;

- Edge $^{0,(2)}(\Gamma, \tau)$ is the set of pairs $\left\{\pi(e), \pi\left(e^{\prime}\right)\right\} \subset \operatorname{Edge}^{0}(\Gamma / \tau)$, where $\left\{e, e^{\prime}\right\} \subset \operatorname{Edge}^{0}(\Gamma)$ is such that $\pi(e), \pi\left(e^{\prime}\right) \notin \operatorname{Edge}^{0,(1)}(\Gamma, \tau)$ and $\Gamma \backslash\left\{e, e^{\prime}\right\}$ is not connected.

Proposition 3.5. Let $(\Gamma, \tau)$ be a real trivalent graph. Then we have

$$
W_{(\Gamma, \tau)}=\left(\bigoplus_{e \in E d g e^{0,(1)}(\Gamma, \tau)} \mathbb{Z} / 2 \mathbb{Z} e\right) \oplus \operatorname{Span}\left\{e+e^{\prime} \mid\left\{e, e^{\prime}\right\} \in \operatorname{Edge}^{0,(2)}(\Gamma, \tau)\right\} .
$$

Proof. Let us denote by $V$ the $\mathbb{Z} / 2 \mathbb{Z}$-vector space on the right hand side of the equality stated in the proposition. Hence we want to show that $W_{(\Gamma, \tau)}=V$.

Step 1: reduction to the case $\tau=\mathrm{Id}$. The map $\pi: \Gamma \rightarrow \Gamma / \tau$ induces a linear map $\tilde{\pi}: \overrightarrow{\Pi_{(\Gamma, \mathrm{Id})}} \rightarrow$ $\overrightarrow{\Pi_{(\Gamma, \tau)}}$. In order to avoid confusion, we denote by $\mu_{\Gamma}$ the map $H_{1}(\Gamma ; \mathbb{Z} / 2 \mathbb{Z}) \times H_{1}(\Gamma ; \mathbb{Z} / 2 \mathbb{Z}) \rightarrow \overrightarrow{\Pi_{(\Gamma, I d)}}$, and we keep the notation $\mu: H_{1}(\Gamma ; \mathbb{Z} / 2 \mathbb{Z}) \times H_{1}(\Gamma ; \mathbb{Z} / 2 \mathbb{Z}) \rightarrow \overrightarrow{\Pi_{(\Gamma, \tau)}}$. Recall that

$$
W_{(\Gamma, \mathrm{Id})}=\left\{w \in \overrightarrow{\Pi_{(\Gamma, \mathrm{Id})}} \mid \forall \alpha, \beta \in H_{1}(\Gamma ; \mathbb{Z} / 2 \mathbb{Z}),\left\langle w, \mu_{\Gamma}(\alpha, \beta)\right\rangle=0\right\} .
$$

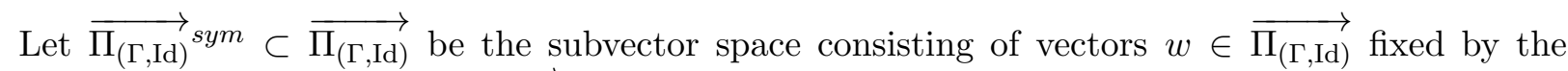
natural map induced by $\tau$ on $\overrightarrow{\Pi_{(\Gamma, I d)}}$. Note that the difference between two real structures above $(\Gamma, \tau)$ can always be expressed as such an element. Given $w \in{\overrightarrow{\Pi_{(\Gamma, \mathrm{Id})}}}_{\text {sym }}$, we write $w=w^{\text {fix }}+w^{\text {even }}$ where $w^{f i x}$ is supported by the edges of $\Gamma$ fixed by $\tau$ and $w^{\text {even }}$ is supported by pairs of edges exchanged by $\tau$. Define the surjective linear map $\bar{\pi}: \overrightarrow{\Pi_{(\Gamma, \mathrm{Id})}}$ sym $\rightarrow \overrightarrow{\Pi_{(\Gamma, \tau)}}$ by $\bar{\pi}(w)=\widetilde{\pi}\left(w^{f i x}\right)+\widetilde{\pi}\left(w^{1}\right)$,

$1 \uparrow$ Recall that a connected real topological surface $(S, \tau)$ is of type I if $S \backslash \mathbb{R} S$ is disconnected, and of type II otherwise. 
where $w^{\text {even }}=w^{1}+\tau\left(w^{1}\right)$ and the supports of $w^{1}$ and $\tau\left(w^{1}\right)$ are disjoint. The vector $w^{1}$ is not unique, however $\tilde{\pi}\left(w^{1}\right)$ does clearly not depend on the particular choice of $w^{1}$.

Let us prove that $W_{(\Gamma, \tau)}=\bar{\pi}\left(W_{(\Gamma, \mathrm{Id})} \cap{\overrightarrow{\Pi_{(\Gamma, \mathrm{Id})}}}_{\text {sym }}\right)$.

Let $w \in{\overrightarrow{\Pi_{(\Gamma, \text { Id })}}}_{\text {sym }}$ and let $(\alpha, \beta) \in H_{1}(\Gamma ; \mathbb{Z} / 2 \mathbb{Z}) \times H_{1}(\Gamma ; \mathbb{Z} / 2 \mathbb{Z})$. Write $\mu_{\Gamma}(\alpha, \beta)=\mu_{\Gamma}^{\text {fix }}+\mu_{\Gamma}^{\text {even }}+$ $\mu_{\Gamma}^{\text {odd }}$, where $\mu_{\Gamma}^{\text {odd }}$ is supported by edges $e \in \alpha \cap \beta$ such that $\tau(e) \notin \alpha \cap \beta$ and $\mu_{\Gamma}^{f i x}$ and $\mu_{\Gamma}^{\text {even }}$ are defined as above (in general, the decomposition is not unique). We obviously have

$$
\left\langle w, \mu_{\Gamma}(\alpha, \beta)\right\rangle=\left\langle w^{f i x}, \mu_{\Gamma}^{f i x}\right\rangle+\left\langle w^{e v e n}, \mu_{\Gamma}^{o d d}\right\rangle .
$$

Similarily, we have $\mu(\alpha, \beta)=\widetilde{\pi}\left(\mu_{\Gamma}^{f i x}\right)+\widetilde{\pi}\left(\mu_{\Gamma}^{\text {odd }}\right)$, and

$$
\langle\bar{\pi}(w), \mu(\alpha, \beta)\rangle=\left\langle\bar{\pi}\left(w^{f i x}\right), \widetilde{\pi}\left(\mu_{\Gamma}^{f i x}\right)\right\rangle+\left\langle\bar{\pi}\left(w^{e v e n}\right), \widetilde{\pi}\left(\mu_{\Gamma}^{o d d}\right)\right\rangle .
$$

Thus $\left\langle w, \mu_{\Gamma}(\alpha, \beta)\right\rangle=\langle\bar{\pi}(w), \mu(\alpha, \beta)\rangle$, which implies that $W_{\Gamma, \tau}=\bar{\pi}\left(W_{\Gamma, \text { Id }} \cap{\overrightarrow{\Pi_{(\Gamma, I d)}}}_{\text {sym }}\right)$ as announced.

Assuming that Proposition 3.5 is known in the case of $\tau=\mathrm{Id}$ and computing $W_{(\Gamma, \mathrm{Id})} \cap{\overrightarrow{\Pi_{(\Gamma, \mathrm{Id})}}}_{\text {sym }}$, it is easy to see that it remains to show that

$$
\operatorname{Edge}^{0,(1)}(\Gamma, \tau)=\pi\left(\operatorname{Edge}^{0,(1)}(\Gamma, \mathrm{Id})\right) \bigcup \pi\left(\left\{\left\{e, e^{\prime}\right\} \subset \operatorname{Edge}^{0,(2)}(\Gamma, \mathrm{Id}) \mid \tau(e)=e^{\prime} \text {, and } \tau\left(e^{\prime}\right)=e\right\}\right)
$$

and

$$
\begin{aligned}
\operatorname{Edge}^{0,(2)}(\Gamma, \tau)= & \pi\left(\left\{\left\{e, e^{\prime}\right\} \subset \operatorname{Edge}^{0,(2)}(\Gamma, \mathrm{Id}) \mid \tau(e) \neq e^{\prime}\right\}\right) \\
= & \pi\left(\left\{\left\{e, e^{\prime}\right\} \subset \operatorname{Edge}^{0,(2)}(\Gamma, \mathrm{Id}) \mid \tau(e)=e \text { and } \tau\left(e^{\prime}\right)=e^{\prime}\right\}\right) \\
& \cup \pi\left(\left\{\left\{e, e^{\prime}\right\} \subset \operatorname{Edge}^{0,(2)}(\Gamma, \mathrm{Id}) \mid \tau(e) \neq e, \tau\left(e^{\prime}\right) \neq e^{\prime} \text { and } \tau(e) \neq e^{\prime}\right\}\right) .
\end{aligned}
$$

These equalities follows from the fact that the quotient map $\pi: \Gamma \rightarrow \Gamma / \tau$ induces a surjective map $\pi_{*}: H_{1}(\Gamma ; \mathbb{Z} / 2 \mathbb{Z}) \rightarrow H_{1}(\Gamma / \tau ; \mathbb{Z} / 2 \mathbb{Z})$.

So from now on, we assume that $\tau=\mathrm{Id}$, and we use the shorter notation

$$
W_{\Gamma}=W_{(\Gamma, \tau)}, \quad \operatorname{Edge}^{0,(1)}(\Gamma)=\operatorname{Edge}^{0,(1)}(\Gamma, \mathrm{Id}), \quad \text { and } \quad \operatorname{Edge}^{0,(2)}(\Gamma)=\operatorname{Edge}^{0,(2)}(\Gamma, \mathrm{Id}) .
$$

Step 2: $V$ is contained in $W_{\Gamma}$. An edge in Edge ${ }^{0,(1)}(\Gamma)$ is not contained in the support of any cycle in $H_{1}(\Gamma ; \mathbb{Z} / 2 \mathbb{Z})$. In particular we have

$$
\bigoplus_{e \in \operatorname{Edge}^{0,(1)}(\Gamma)} \mathbb{Z} / 2 \mathbb{Z} e \subset W_{\Gamma}
$$

We claim that a pair of edges $\left\{e, e^{\prime}\right\} \subset \operatorname{Edge}^{0}(\Gamma)$ is contained in $\operatorname{Edge}^{0,(2)}(\Gamma)$ if and only if the two following conditions are satisfied:

1. there exists a cycle $\gamma \in H_{1}(\Gamma ; \mathbb{Z} / 2 \mathbb{Z})$ containing both $e$ and $e^{\prime}$;

2. for any theta subgraph $\Theta$ of $\Gamma$ containing $e$ and $e^{\prime}$, the graph $\Theta \backslash\left\{e, e^{\prime}\right\}$ is not connected, see Figure $7 \mathrm{a}$ and $\mathrm{b}$. 


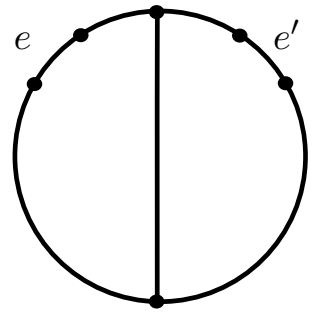

a) $\Theta \backslash\left\{e, e^{\prime}\right\}$ connected

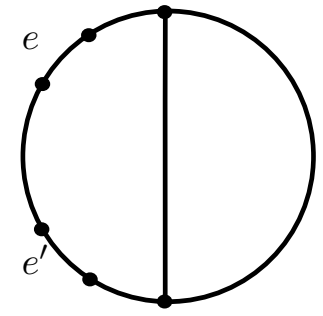

b) $\Theta \backslash\left\{e, e^{\prime}\right\}$ disconnected

Figure 7:

Indeed, condition (1) is equivalent to the fact that neither $e$ nor $e^{\prime}$ is in $\operatorname{Edge}^{0,(1)}(\Gamma)$. Next, a pair $\left\{e, e^{\prime}\right\}$ satisfying condition (1) is in $\operatorname{Edge}^{0,(2)}(\Gamma)$ if and only if any path in $\Gamma \backslash\{e\}$ joining the two vertices adjacent to $e$ contains $e^{\prime}$. This is equivalent to condition (2). have

It follows from the above claim that for any $\left\{e, e^{\prime}\right\} \in \operatorname{Edge}^{0,(2)}(\Gamma)$ and any $\gamma \in H_{1}(\Gamma ; \mathbb{Z} / 2 \mathbb{Z})$, we

$$
e \subset \gamma \Longleftrightarrow e^{\prime} \subset \gamma
$$

This implies that

$$
\operatorname{Span}\left\{e+e^{\prime} \mid\left\{e, e^{\prime}\right\} \in \operatorname{Edge}^{0,(2)}(\Gamma)\right\} \subset W_{\Gamma}
$$

and so $V \subset W_{\Gamma}$.

We define the following vector subspace of $\overrightarrow{\Pi_{(\Gamma, \tau)}}$ :

$$
U=\left(\bigoplus_{e \in \mathrm{Edge}^{0,(1)}(\Gamma, \tau)} \mathbb{Z} / 2 \mathbb{Z} e\right) \oplus \operatorname{Span}\left\{e \mid \exists e^{\prime} \text { with }\left\{e, e^{\prime}\right\} \in \operatorname{Edge}^{0,(2)}(\Gamma, \tau)\right\}
$$

Step 3: $W_{\Gamma}$ is contained in $U$. Let $e \notin U$. It means exactly that $e \in \operatorname{Edge}^{0}(\Gamma) \backslash \operatorname{Edge}^{0,(1)}(\Gamma)$ and that $\Gamma \backslash\left\{e, e^{\prime}\right\}$ is connected for any $e^{\prime} \in \operatorname{Edge}^{0}(\Gamma) \backslash \operatorname{Edge}^{0,(1)}(\Gamma)$. Denote by $v$ and $v^{\prime}$ the two vertices of $\Gamma$ adjacent to $e$. By Menger's Theorem [BR12, Theorem 3.6.11], there exist two paths $c_{1}$ and $c_{2}$ in $\Gamma \backslash\{e\}$ joining $v$ and $v^{\prime}$, and whose intersection is reduced to $\left\{v, v^{\prime}\right\}$. Hence $e$ is the only edge common to the two cycles $c_{1} \cup e$ and $c_{2} \cup e$ in $H_{1}(\Gamma ; \mathbb{Z} / 2 \mathbb{Z})$. By definition of $W_{\Gamma}$, we have $\langle w, e\rangle=0$ for any $w \in W_{\Gamma}$, and so $e \notin W_{\Gamma}$. We conclude that $W_{\Gamma} \subset U$.

Step 4: $W_{\Gamma}$ is contained in $V$. By Step 3 above, an element $\widetilde{w}$ of $W_{\Gamma}$ can be written in the (non-unique) form

$$
\widetilde{w}=w_{0}+e_{1}+\ldots+e_{k}+\left(e_{k+1}+e_{k+1}^{\prime}\right)+\ldots+\left(e_{m}+e_{m}^{\prime}\right),
$$

where

$$
\text { - } w_{0} \in \bigoplus_{e \in \operatorname{Edge}^{0,(1)}(\Gamma)} \mathbb{Z} / 2 \mathbb{Z} e
$$

- $\left\{e_{i}, e_{i}^{\prime}\right\} \in \operatorname{Edge}^{0,(2)}(\Gamma)$ if $i \in\{k+1, \ldots, m\}$;

- for any $i \in\{1, \ldots, k\}$, there exists an edge $e_{i}^{\prime}$ of $\Gamma$ such that $\left\{e_{i}, e_{i}^{\prime}\right\} \in \operatorname{Edge}^{0,(2)}(\Gamma)$;

- $e_{i} \neq e_{j}$ and $\left\{e_{i}, e_{j}\right\} \notin \operatorname{Edge}^{0,(2)}(\Gamma)$ for any pair $\{i, j\} \subset\{1, \ldots, k\}$. 
Recall that $V$ is a sub-vector space of $W_{\Gamma}$ by Step 2, hence $w=w_{0}+e_{1}+\ldots+e_{k}$ is an element of $W_{\Gamma}$. If $k=0$, then $w$ is in $V$, and so is $\widetilde{w}$. Assume now that $k>0$. In this case $w$ is an element of $W_{\Gamma}$ such that for any edge $e \in \operatorname{Edge}^{0}(\Gamma) \backslash \operatorname{Edge}^{0,(1)}(\Gamma)$ with $\langle w, e\rangle=1$, we have $\left\langle w, e^{\prime}\right\rangle=0$ for any edge $e^{\prime}$ with $\left\{e, e^{\prime}\right\} \in \operatorname{Edge}^{0,(2)}(\Gamma)$. The rest of the proof consists in proving by contradiction that such an element cannot exist.

Let $\alpha \in H_{1}(\Gamma ; \mathbb{Z} / 2 \mathbb{Z})$ be a cycle containing an edge in the support of $w$. Let us denote by $e_{1}, \ldots, e_{k}$ all edges belonging to the intersection of the support of $w$ and $\alpha$, enumerated in a cyclic order induced by $\alpha$. By definition of $W_{\Gamma}$, we have $\langle w, \mu(\alpha, \alpha)\rangle=0$, i.e. $k$ is even. Let us also denote by $u_{i}$ the connected component of $\alpha \backslash\left\{e_{1}, \ldots, e_{k}\right\}$ adjacent to the edges $e_{i}$ and $e_{i+1}$ (where the indices are taken modulo $k$ ). By assumption on $w$, for each $i$ there exists a path $c_{i}$ in $\Gamma$ joining the two connected components of $\alpha \backslash\left\{e_{i}, e_{i+1}\right\}$, and such that $c_{i} \cap \alpha$ is reduced to the two endpoints of $c_{i}$. Denote by $\sigma(i) \in \mathbb{Z} / k \mathbb{Z}$ the integer such that $u_{i} \cup u_{\sigma(i)}$ contains the two endpoints of $c_{i}$. Hence $\sigma: \mathbb{Z} / k \mathbb{Z} \rightarrow \mathbb{Z} / k \mathbb{Z}$ is an involution with no fixed points. The two endpoints of $c_{i}$ divide $\alpha$ into two connected components. Let $u$ be one of these latter. Since $w \in W_{\Gamma}$, we have $\left.\left\langle w, \mu\left(\alpha, u \cup c_{i}\right)\right)\right\rangle=0$, that is to say $\sigma(i)=i$ mod 2 (see Figure 8a). Furthermore, we claim that for any $j$, we have

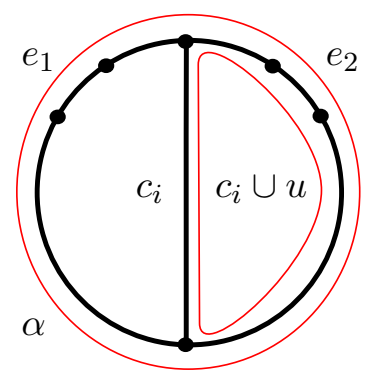

a)

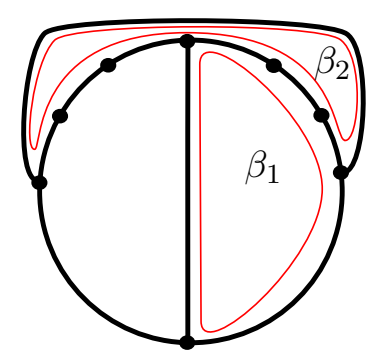

b)

Figure 8:

$$
j \in\{i+1, \ldots, \sigma(i)-1\} \Longleftrightarrow \sigma(j) \in\{i+1, \ldots, \sigma(i)-1\} .
$$

It is enough to prove the claim in the case when $j=i+1$. If $\sigma(i+1) \notin\{i+1, \ldots, \sigma(i)-1\}$, one easily constructs two cycles $\beta_{1}$ and $\beta_{2}$ in $H_{1}(\Gamma ; \mathbb{Z} / 2 \mathbb{Z})$ such that $\left.\left\langle w, \mu\left(\beta_{1}, \beta_{2}\right)\right)\right\rangle=1$ (see Figure $8 \mathrm{~b}$ ), which contradicts that $w \in W_{\Gamma}$.

Hence the map $\sigma$ induces an involution on $\{2, \ldots, \sigma(1)-1\}$ with no fixed points. However the cardinal of this latter set is odd, so such a fixed-point free involution cannot exist.

Recall that a graph is said to be $k$-edge connected if it remains connected after removing any set of $l<k$ edges.

Corollary 3.6. Let $(\Gamma, \tau)$ be a real trivalent graph. If $\Gamma / \tau$ is 3-edge connected, then $W_{(\Gamma, \tau)}$ is the trivial vector space. In particular there exists at most one real structure $\tau_{\Gamma}$ above $(\Gamma, \tau)$ for which $\left(S_{\Gamma}, \tau_{\Gamma}\right)$ is maximal.

\section{Haas' Theorem}

Here we explain how the results from the previous section specialise to Haas' Theorem in the particular case of non-singular tropical curves in $\mathbb{R}^{2}$. We first give in Section 4.A a tropical formulation of Viro's combinatorial patchworking, and state Haas' Theorem classifying combinatorial patchworkings producing $M$-curves. We prove this latter in Section 4.B. 
We assume that the reader has a certain acquaintance with tropical geometry. We refer to [BIMS15] for an introduction to tropical geometry at the level needed here, as well as a more detailed exposition of combinatorial patchworking and Haas' Theorem.

\section{A. Combinatorial patchworking and Haas' Theorem}

Here we present the reformulation of the particular case of unimodular combinatorial patchworking in terms of twist-admissible sets of edges of a non-singular plane tropical curve given in [BIMS15]. If $e$ is an edge of a tropical curve in $\mathbb{R}^{2}$, we denote by $\left(x_{e}, y_{e}\right) \in \mathbb{Z}^{2}$ a primitive direction vector of the line supporting $e$ (note that $\left(x_{e}, y_{e}\right)$ is well defined up to sign, however this does not play a role in what follows).

Definition 4.1. Let $C$ be a non-singular tropical curve in $\mathbb{R}^{2}$. A subset $T$ of $\operatorname{Edge}^{0}(C)$ is called twist-admissible if for any cycle $\gamma$ of $C$, we have

$$
\sum_{e \in \gamma \cap T}\left(x_{e}, y_{e}\right)=0 \bmod 2 .
$$

Given $T$ a twist-admissible subset of edges of a non-singular tropical curve $C$ in $\mathbb{R}^{2}$, perform the following operations:

(a) at each vertex of $C$, draw three arcs as depicted in Figure 9a;

(b) for each edge $e \in \operatorname{Edge}^{0}(C)$ adjacent to the vertices $v$ and $v^{\prime}$, join the two corresponding arcs at $v$ to the corresponding ones for $v^{\prime}$ in the following way: if $e \notin T$, then join these arcs as depicted in Figure 9b; if $e \in T$, then join these arcs as depicted in Figure 9c; denote by $\mathcal{P}$ the obtained collection of arcs;

(c) choose arbitrarily an arc of $\mathcal{P}$ and a pair of signs for it;

(d) associate pairs of signs to all arcs of $\mathcal{P}$ using the following rule: given $e \in \operatorname{Edge}(C)$ the pairs of signs of the two arcs of $\mathcal{P}$ corresponding to $e$ differ by a factor $\left((-1)^{x_{e}},(-1)^{y_{e}}\right)$, see Figure $9 \mathrm{~b}$ and c; (Note that the compatibility condition (4.2) precisely means that this rule is consistent.)

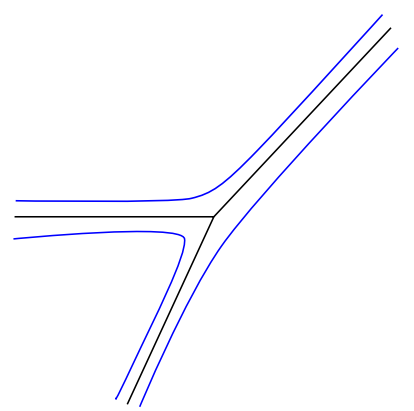

a)

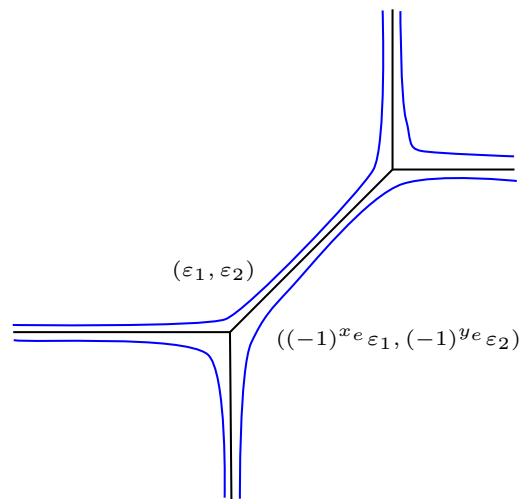

b) $e \notin T$

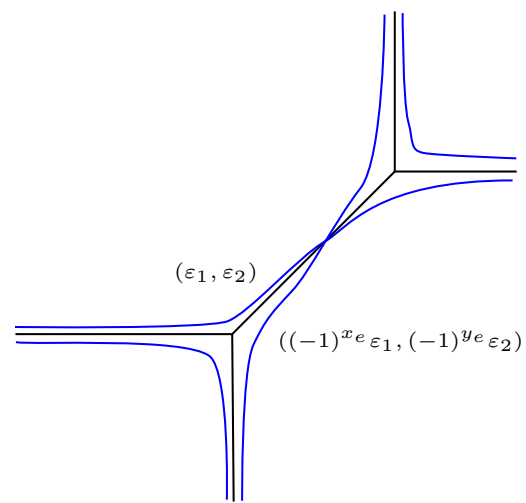

c) $e \in T$

Figure 9: A patchworking of a non-singular tropical curve 
(e) map each arc $A$ of $\mathcal{P}$ to $\left(\mathbb{R}^{\times}\right)^{2}$ by $(x, y) \mapsto\left(\varepsilon_{1} \exp (x), \varepsilon_{2} \exp (y)\right)$, where $\left(\varepsilon_{1}, \varepsilon_{2}\right)$ is the pair of signs associated to $A$. Denote by $C_{T}$ the curve in $\left(\mathbb{R}^{\times}\right)^{2}$ which is the union of these images over all arcs of $\mathcal{P}$.

Note that all possible choices at step (c) above produce the same curve $C_{T}$ up to the action of $(\mathbb{Z} / 2 \mathbb{Z})^{2}$ by axial symmetries $(z, w) \mapsto( \pm z, \pm w)$. Viro combinatorial patchworking Theorem [Vir01] may be reformulated in terms of twist-admissible sets as follows.

Theorem 4.2. (Viro) Let $T$ be a twist-admissible subset of edges of a non-singular tropical curve $C$ in $\mathbb{R}^{2}$. Then there exists a real algebraic curve in $\left(\mathbb{C}^{\times}\right)^{2}$ with the same Newton polygon as $C$, and whose real part in $\left(\mathbb{R}^{\times}\right)^{2}$ is isotopic to $C_{T}$.

Remark 4.3. It is possible to produce an equation for the real algebraic curve whose existence is attested by Theorem 4.2, see [BIMS15, Remark 3.9].

Example 4.4. One may choose $T$ to be empty as the empty set clearly satisfies (4.2). This corresponds to Harnack patchworking mentioned in the introduction. The resulting curve corresponds to the construction of simple Harnack curves described in [Mik00] via Harnack distribution of signs, see [IV96]. Furthermore, the isotopy type of $C_{T}$ in $\left(\mathbb{R}^{\times}\right)^{2}$, up to axial symmetries, only depends on the Newton polygon of $C$, see Proposition 4.11.

Example 4.5. Let us consider the non-singular tropical curve $C$ of degree 6 depicted on Figure 10. We equip $C$ with three different twist-admissible collection of edges in Figures 11, 12, and 13. In each case we depict the isotopy types of the real part of the corresponding real algebraic curve in both $\left(\mathbb{R}^{\times}\right)^{2}$ and $\mathbb{R} \mathbb{P}^{2}$. Note that these are the only isotopy types of maximal real sextics in $\mathbb{R P}^{2}$.

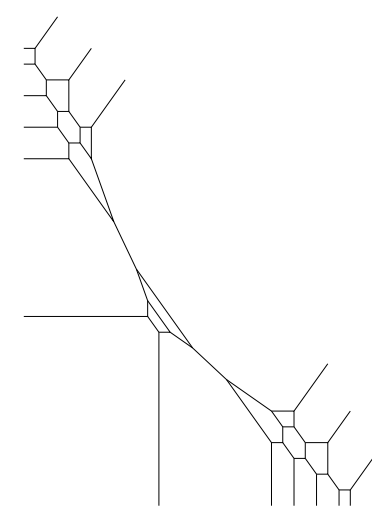

Figure 10: A tropical sextic

Given a non-singular tropical curve $C$, Haas' classified in [Haa97] all twist-admissible sets producing an $M$-curve. At that time the formalism of tropical geometry did not exist yet, and the original formulation of Haas' theorem is dual to the one we present here in Theorem 4.6.

We say that a twist-admissible set of edges $T$ of $C$ is maximal if it satisfies the two following conditions:

1. any cycle in $H_{1}(C ; \mathbb{Z} / 2 \mathbb{Z})$ contains an even number of edges in $T$;

2. for any edge $e \in T$, either $C \backslash e$ is disconnected, or there exists an edge $e^{\prime} \in T$ such that $C \backslash e$ and $C \backslash e^{\prime}$ are connected, but $C \backslash\left(e \cup e^{\prime}\right)$ is disconnected.

Theorem 4.6. (Haas) Let $C$ be a non-singular tropical curve in $\mathbb{R}^{2}$, and let $T$ be a twist-admissible set of edges of $C$. Then a real algebraic curve whose existence is asserted by Theorem 4.2 is maximal if and only if $T$ is maximal. 

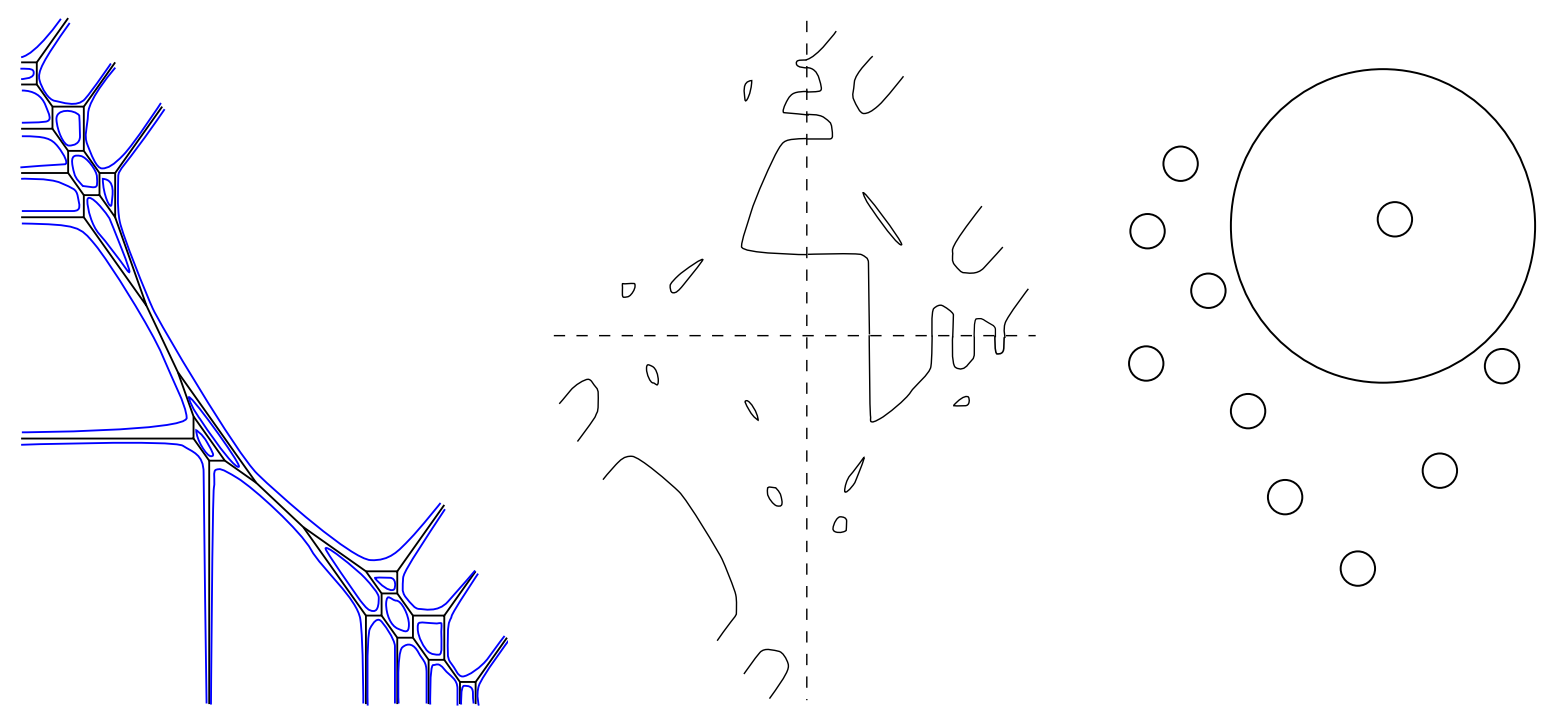

Figure 11: Simple Harnack sextic
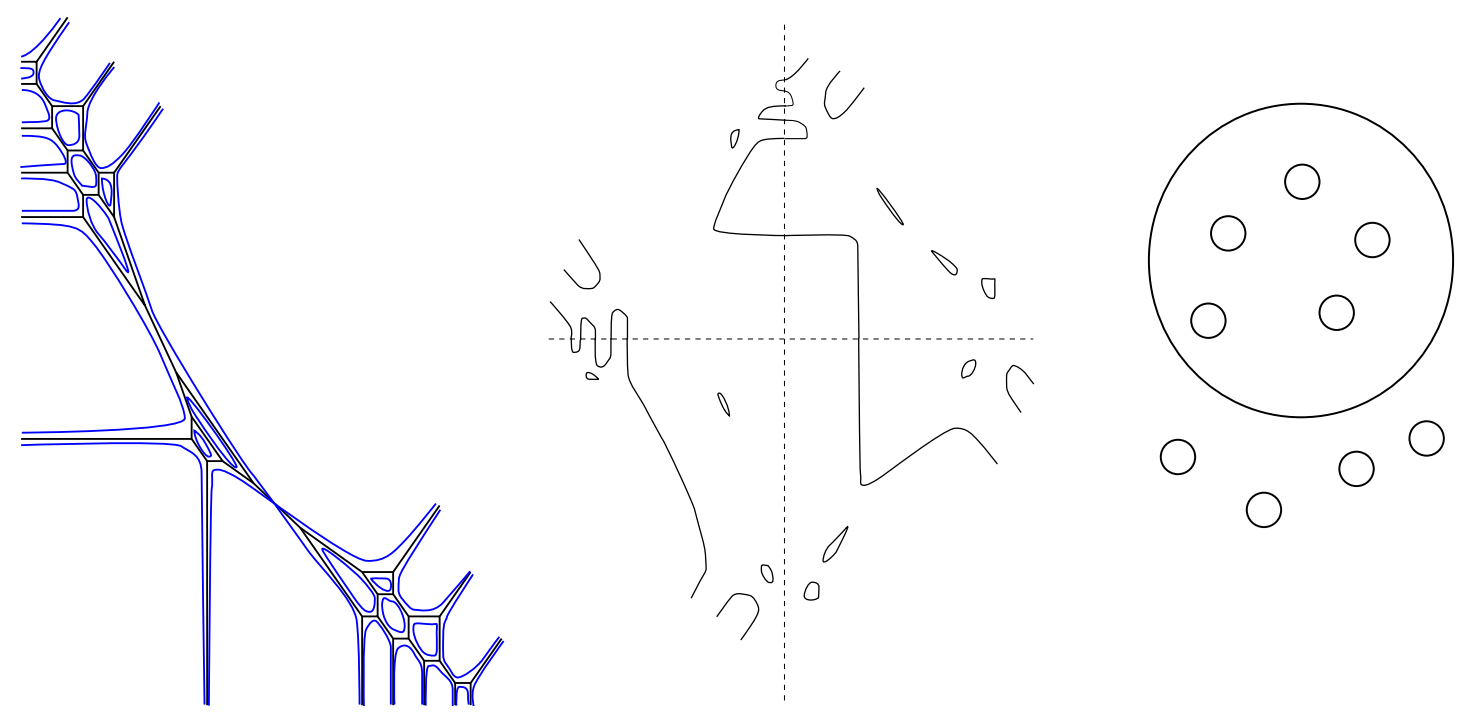

Figure 12: Gudkov's sextic

Example 4.7. The empty collection is maximal, hence we recover the existence, for each non-singular tropical curve, of a canonical maximal patchworking mentioned in the introduction.

\section{B. Proof of Haas' Theorem}

Here we deduce Theorem 4.6 from Theorem 3.2 and Proposition 3.5. First, we introduce some standard notations.

The coordinatewise argument and log maps are defined by:

$$
\begin{aligned}
& \operatorname{Arg}:\left(\mathbb{C}^{\times}\right)^{2} \longrightarrow(\mathbb{R} / 2 \pi \mathbb{Z})^{2} \quad \text { and } \quad \log :\left(\mathbb{C}^{\times}\right)^{2} \longrightarrow \mathbb{R}^{2} \\
& (z, w) \longmapsto(\arg (z), \arg (w)) \quad \text { and } \quad(z, w) \longmapsto(\log |z|, \log |w|)
\end{aligned}
$$

Note that the image of the map Arg is canonically identified with any fiber of the map Log. We also 

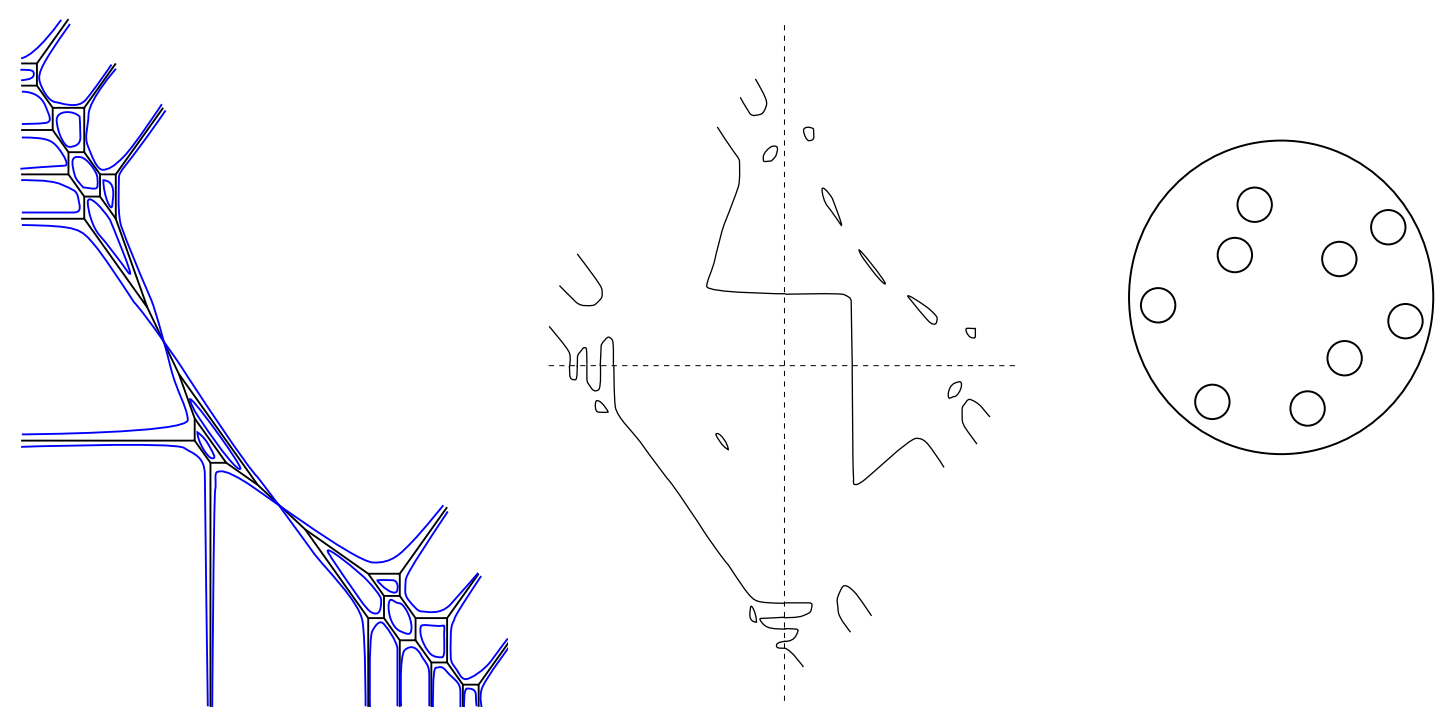

Figure 13: Hilbert's sextic

define the following self-diffeomorphism of $\left(\mathbb{C}^{\times}\right)^{2}$ fot $t>1$

$$
\begin{aligned}
H_{t}:\left(\mathbb{C}^{\times}\right)^{2} & \longrightarrow\left(\mathbb{C}^{\times}\right)^{2} \\
(z, w) & \longmapsto\left(|z|^{\frac{1}{\log (t)}} \frac{z}{|z|},|w|^{\frac{1}{\log (t)}} \frac{w}{|w|}\right) .
\end{aligned}
$$

Given a complex polynomial $P(z, w)=\sum a_{i, j} z^{i} w^{j}$ and $\Delta \subset \mathbb{R}^{2}$, we define

$$
P^{\Delta}(z, w)=\sum_{(i, j) \in \Delta} a_{i, j} z^{i} w^{j}
$$

The (closed) coamoeba of the algebraic curve $X$ with equation $P(z, w)=0$, denoted by $\mathcal{C} \mathcal{A}(P)$, is defined as the topological closure in $(\mathbb{R} / 2 \pi \mathbb{Z})^{2}$ of the set $\operatorname{Arg}(X)$.

Example 4.8. If $P(z, w)$ is a real binomial whose Newton segment $\Delta(P)$ has integer length 1 , then the real part of the real algebraic curve defined by $P(z, w)$ intersects two quadrants $Q_{1}$ and $Q_{2}$ of $\left(\mathbb{R}^{\times}\right)^{2}$. If $u$ denotes a primitive integer vector normal to $\Delta(P)$, then the coamoeba $\mathcal{C} \mathcal{A}(P)$ is the geodesic in $(\mathbb{R} / 2 \pi \mathbb{Z})^{2}$ with direction $u$ and passing through $\operatorname{Arg}\left(Q_{1}\right)$ and $\operatorname{Arg}\left(Q_{2}\right)$. On Figure 14 a we depicted the coamoeba of a line given by the equation $z+a w=0$ with $a>0$. It joins the points $(0, \pi)$ and $(\pi, 0)$.

Example 4.9. (See [Mik05, Proposition 6.11 and Lemma 8.19]) If $P(z, w)$ is a real trinomial whose Newton triangle $\Delta(P)$ has Euclidean area $\frac{1}{2}$, then the real part of the real algebraic curve defined by $P(z, w)$ intersects three quadrants $Q_{1}, Q_{2}$, and $Q_{3}$ of $\left(\mathbb{R}^{\times}\right)^{2}$. If $u_{1}, u_{2}$, and $u_{3}$ denote primitive integer vectors normal to the three edges of $\Delta(P)$, then the coamoeba $\mathcal{C} \mathcal{A}(P)$ is the union of the two triangles with vertices $\operatorname{Arg}\left(Q_{1}\right), \operatorname{Arg}\left(Q_{2}\right)$, and $\operatorname{Arg}\left(Q_{3}\right)$, and whose sides are geodesics with direction $u_{1}, u_{2}$, and $u_{3}$. In particular $\mathcal{C} \mathcal{A}(P)$ is a (degenerate) pair of pants. On Figure $14 \mathrm{~b}$ we represented the coamoeba of a line given by the equation $z+a w+b=0$ with $a>0$ and $b<0$.

Recall (see for example [BIMS15, Section 2.2]) that to any tropical curve $C$ in $\mathbb{R}^{2}$ is associated a dual subdivision of its Newton polygon. We denote respectively by $\Delta_{v}$ and $\Delta_{e}$ the polygon dual to the vertex $v$ and the edge $e$ of $C$. Next statement is proved in [Mik05, Section 6]. 


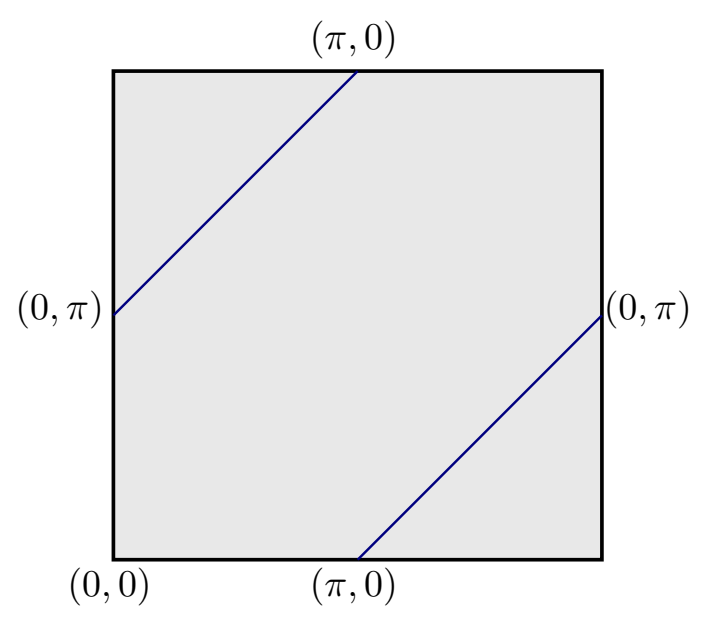

a) Coamoeba of a real line defined by $z+a w$ with $a>0$

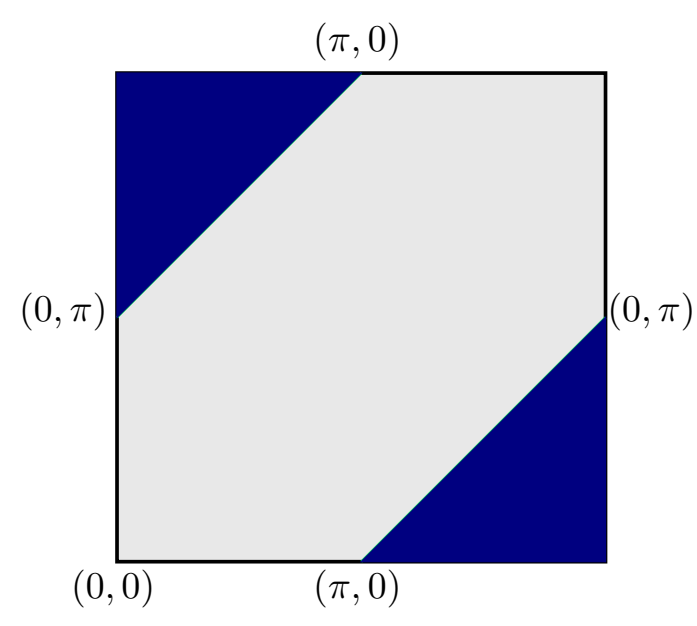

b) Coamoeba of a real line defined by $z+a w+b$ with $a>0$ and $b<0$

Figure 14:

Theorem 4.10. (Mikhalkin) Let $C$ be a tropical curve in $\mathbb{R}^{2}$ defined by some tropical polynomial " $\sum b_{i, j} x^{i} y^{j}$ ". Then given a collection $a_{i, j}$ of non-zero complex numbers, the image by $H_{t}$ of the algebraic curve in $\left(\mathbb{C}^{\times}\right)^{2}$ defined by the complex polynomial

$$
P_{t}(z, w)=\sum a_{i, j} t^{-b_{i, j}} z^{i} w^{j}
$$

converges when $t \rightarrow+\infty$, for the Hausdorff metric on compact subsets of $\left(\mathbb{C}^{\times}\right)^{2}$, to a subset $V_{\infty}$ that can be described as follows:

- $\log \left(V_{\infty}\right)=C$;

- for any vertex $v$ of $C$, we have $\log ^{-1}(v) \cap V_{\infty}=\mathcal{C} \mathcal{A}\left(P_{1}^{\Delta_{v}}\right)$;

- for each edge e of $C$, we have $\log ^{-1}(e) \cap V_{\infty}=e \times \mathcal{C} \mathcal{A}\left(P_{1}^{\Delta_{e}}\right)$.

If furthermore all the $a_{i, j}$ 's are real numbers, then the real part of the real algebraic curve defined by $P_{t}(z, w)$ converges when $t \rightarrow+\infty$, for the Hausdorff metric on compact subsets of $\left(\mathbb{R}^{\times}\right)^{2}$, to $V_{\infty} \cap\left(\mathbb{R}^{\times}\right)^{2}$.

Note that one can recover Theorem 4.2 by combining Theorem 4.10 together with Examples 4.8 and 4.9. In particular, an equation of a simple Harnack curve is given by next proposition. We define the function $\varepsilon: \mathbb{Z}^{2} \rightarrow\{ \pm 1\}$ by $\varepsilon(i, j)=1$ if both $i$ and $j$ are even, and by $\varepsilon(i, j)=-1$ otherwise. Recall that the Viro's patchworking construction and the definition of a twist admissible set of edges of a non-singular tropical curve are given in Section 4.A.

Proposition 4.11. (Itenberg, see [IV96] or [BIMS15, Remark 3.9]) Let C be a non-singular tropical curve in $\mathbb{R}^{2}$ defined by some tropical polynomial " $\sum b_{i, j} x^{i} y^{j}$ ", and let

$$
P_{t}(z, w)=\sum \varepsilon(i, j) t^{-b_{i, j}} z^{i} w^{j}, \quad V_{\infty}=\lim _{t \rightarrow+\infty} H_{t}\left(\left\{P_{t}=0\right\}\right) .
$$

Then up to axial symmetries, the real part of $V_{\infty}$ is isotopic in $\left(\mathbb{R}^{\times}\right)^{2}$ to the curve $C_{\emptyset}$ constructed out of $C$ and the empty twist admissible set. In particular, the real algebraic curve defined by $P_{t}(z, w)$ with $t$ large enough is maximal. Furthermore, the isotopy type of its real part in $\left(\mathbb{R}^{\times}\right)^{2}$, up to axial symmetries, only depends on the Newton polygon of $C$. 
We are now ready to deduce Haas' Theorem from what is discussed above.

Proof of Theorem 4.6. Let $C$ be a non-singular tropical curve in $\mathbb{R}^{2}$ defined by the tropical polynomial " $\sum b_{i, j} x^{i} y^{j}$ ", and let $\nu: \mathbb{Z}^{2} \rightarrow\{ \pm 1\}$ be some function. We define

$$
P_{t}(z, w)=\sum \varepsilon(i, j) t^{-b_{i, j}} z^{i} w^{j}, \quad V_{\infty}^{0}=\lim _{t \rightarrow+\infty} H_{t}\left(\left\{P_{t}=0\right\}\right),
$$

and

$$
R_{t}(z, w)=\sum \nu(i, j) t^{-b_{i, j}} z^{i} w^{j}, \quad V_{\infty}=\lim _{t \rightarrow+\infty} H_{t}\left(\left\{R_{t}=0\right\}\right)
$$

We equipped both $V_{\infty}$ and $V_{\infty}^{0}$ with the real structure coming from the restriction of the complex conjugation on $\left(\mathbb{C}^{\times}\right)^{2}$. Compactifying $C$ by gluing a point to each unbounded edge $C$, we obtain a graph $\Gamma$. The map Log : $V_{\infty}^{0} \rightarrow C$ induces a pair of pants decomposition of the topological surface $V_{\infty}^{0}$. To each unbounded edge of $C$ corresponds an unbounded cylinder of $V_{\infty}^{0}$. A surface $S_{\Gamma}$ as in Section 2.A is obtained by gluing a disk to each such unbounded cylinder of $V_{\infty}^{0}$, and we have the identities

$$
S_{v}=\log ^{-1}(v) \cap V_{\infty}^{0} \quad \forall v \in \operatorname{Vert}(C), \quad \text { and } \quad S_{e}=\log ^{-1}(e) \cap V_{\infty}^{0} \quad \forall e \in \operatorname{Edge}(C),
$$

up to considering degenerate pairs of pants for $S_{v}$ instead of usual ones in the construction of $S_{\Gamma}$ in Section 2.A. All previous definitions and results are easily seen to hold with this benign substitution. Furthermore, the real structure on $V_{\infty}^{0}$ induces a real structure $\tau_{\Gamma, 1}$ above the real graph $(\Gamma, \mathrm{Id})$.

Since $C$ in non-singular, for any vertex $v$ of $C$ there exists $\left(\mu_{1}, \mu_{2}\right) \in\{ \pm 1\}^{2}$ such that

$$
R_{t}^{\Delta_{v}}\left(\mu_{1} z, \mu_{2} w\right)= \pm P_{t}^{\Delta_{v}}(z, w)
$$

In particular, the map $s_{v}:(z, w) \mapsto\left(\mu_{1} z, \mu_{2} w\right)$ induces a real homeomorphism (i.e. commuting with real structures)

$$
\zeta_{v}: \log ^{-1}(v) \cap V_{\infty} \rightarrow S_{v} .
$$

Let $\zeta: V_{\infty} \rightarrow V_{\infty}^{0}$ be a (not necessarily real) homeomorphism restricting to $\zeta_{v}$ on $\log ^{-1}(v) \cap V_{\infty}$ for each vertex $v$ of $C$. Note that $\zeta$ is well defined up to isotopy and conjugation by a finite product of Dehn twists along cylinders $S_{e}$ with $e \in \operatorname{Edge}^{0}(\Gamma)$. Furthermore, given an edge $e \in \operatorname{Edge}^{0}(\Gamma)$ adjacent to two vertices $v$ and $v^{\prime}$, the two maps $s_{v}$ and $s_{v^{\prime}}$ coincide if and only if, up to an isotopy restricting on the identity on $\partial S_{e}$, the restriction of $\zeta$ to $\log ^{-1}(e) \cap V_{\infty}$ is a real map. The real structure on $V_{\infty}$ induces, via the map $\zeta$, a real structure on $V_{\infty}^{0}$, which in its turn induces a real structure $\tau_{\Gamma, 2}$ above the real graph $\left(\Gamma\right.$, Id). Let $T$ be the set of edges for which $\left.\zeta\right|_{\log ^{-1}(e) \cap V_{\infty}}$ is not a real map. By Lemma 2.9 , we have

$$
\tau_{\Gamma, 2}-\tau_{\Gamma, 1}=\sum_{e \in T} e
$$

On the other hand, by [BIMS15, Theorem 3.4 and Remark 3.9], the set $T$ is twist-admissible and the real part of the algebraic curve in $\left(\mathbb{C}^{\times}\right)^{2}$ with equation $R_{t}(z, w)=0$ is isotopic in $\left(\mathbb{R}^{\times}\right)^{2}$ to the curve $C_{T}$ for $t$ large enough. Hence by Corollary 3.4 and Proposition 3.5 applied to $\tau_{\Gamma, 1}$ and $\tau_{\Gamma, 2}$, the real algebraic curve in $R_{t}(z, w)=0$ for $t$ large enough is maximal if and only if $T$ is maximal.

To finish the proof of Haas' Theorem, it is enough to notice that for any set $T$ of twist-admissible edges of $C$, there exists a function $\nu: \mathbb{Z}^{2} \rightarrow\{ \pm 1\}$ as above such that the real part of the algebraic curve in $\left(\mathbb{C}^{\times}\right)^{2}$ with equation

$$
\sum \nu(i, j) t^{-b_{i, j}} z^{i} w^{j}=0
$$

is isotopic in $\left(\mathbb{R}^{\times}\right)^{2}$ to the curve $C_{T}$ for $t$ large enough, see [BIMS15, Remark 3.9]. 


\section{References}

[BIMS15] E. Brugallé, I. Itenberg, G. Mikhalkin, and K. Shaw, Brief introduction to tropical geometry. In: Proceedings of the Gökova Geometry-Topology Conference 2014, pp. 1-75, Gökova Geometry/Topology Conference (GGT), Gökova, 2015. MR-3381439

[BR90] R. Benedetti and J.-J. Risler, Real algebraic and semi-algebraic sets, Hermann, Paris, 1990. MR-1070358

[BR12] R. Balakrishnan and K. Ranganathan, A Textbook of Graph Theory, second edition, Springer, New York, 2012. MR-2977757

[Bru06] E. Brugallé, Real plane algebraic curves with asymptotically maximal number of even ovals, Duke Math. J. 131 (2006), no. 3, 575-587. MR-2219251

[Haa95] B. Haas, Les multilucarnes: nouveaux contre-exemples à la conjecture de Ragsdale, C. R. Acad. Sci. Paris Sér. I Math. 320 (1995), no. 12, 1507-1512. MR-1340062

[Haa97] B. Haas, Real algebraic curves and combinatorial constructions. Thèse doctorale, Université de Strasbourg, 1997.

[IKMZ16] I. Itenberg, L. Katzarkov, G. Mikhalkin, and I. Zharkov, Tropical homology, preprint, 2016. arXiv:1604.01838

[Ite93] I. Itenberg, Contre-exemples à la conjecture de Ragsdale, C. R. Acad. Sci. Paris Sér. I Math. 317 (1993), no. 3, 277-282. MR-1233426

[IV96] I. Itenberg and O. Ya. Viro, Patchworking algebraic curves disproves the Ragsdale conjecture, Math. Intelligencer 18 (1996), no. 4, 19-28. MR-1413249

[Kle76] F. Klein, Ueber eine neue Art von Riemann'schen Flächen, Math. Ann. 10 (1876), no. 3, 398-416. MR-1509893

[Mik00] G. Mikhalkin, Real algebraic curves, the moment map and amoebas, Ann. of Math. (2) 151 (2000), no. 1, 309-326. MR-1745011

[Mik05] G. Mikhalkin, Enumerative tropical algebraic geometry in $\mathbb{R}^{2}$, J. Amer. Math. Soc. 18 (2005), no. 2, 313-377. MR-2137980

[Pet33] I. G. Petrovsky, Sur la topologie des courbes réelles algébriques, C. R. Acad. Sci. Paris Sér. I Math. 197 (1933), 1270-1272.

[Rag06] V. Ragsdale, On the arrangement of the real branches of plane algebraic curves, Am. J. Math. 28 (1906), no. 4, 377-404. MR-1505999

[Vir84] O. Ya. Viro, Gluing of plane real algebraic curves and constructions of curves of degrees 6 and 7. In: Topology (Leningrad, 1982), pp. 187-200, Lecture Notes in Math., vol. 1060, Springer, Berlin, 1984. MR-0770238

[Vir01] O. Ya. Viro, Dequantization of real algebraic geometry on logarithmic paper. In: European Congress of Mathematics, Vol. I (Barcelona, 2000), pp. 135-146, Progr. Math., vol. 201, Birkhäuser, Basel, 2001. MR-1905317 